\title{
STRATIFICATION OF THE FOURTH SECANT VARIETY OF VERONESE VARIETIES VIA THE SYMMETRIC RANK
}

\author{
EDOARDO BALLICO, ALESSANDRA BERNARDI
}

\begin{abstract}
If $X \subset \mathbb{P}^{n}$ is a projective non degenerate variety, the $X$-rank of a point $P \in \mathbb{P}^{n}$ is defined to be the minimum integer $r$ such that $P$ belongs to the span of $r$ points of $X$. We describe the complete stratification of the fourth secant variety of any Veronese variety $X$ via the $X$-rank. This result has an equivalent translation in terms either of symmetric tensors or of homogeneous polynomials. It allows to classify all the possible integers $r$ that can occur in the minimal decomposition of a homogeneous polynomial of $X$-border rank 4 (i.e. contained in the fourth secant variety) as a linear combination of powers of linear forms.
\end{abstract}

\section{INTRODUCTION}

Fix integers $m \geq 2$ and $d \geq 2$ and set $n_{m, d}:=\left(\begin{array}{c}m+d \\ m\end{array}\right)-1$. All along this paper the field $\mathbb{K}$ over which all the projective spaces and all the vector spaces will be defined is algebraically closed and of characteristic 0 . Let $\nu_{m, d}: \mathbb{P}^{m} \hookrightarrow \mathbb{P}^{n_{m, d}}$ be the order $d$ Veronese embedding of $\mathbb{P}^{m}$ defined by the sections of the sheaf $\mathcal{O}_{\mathbb{P}^{m}}(d)$. Set:

$$
X_{m, d}:=\nu_{m, d}\left(\mathbb{P}^{m}\right) .
$$

We often set $X:=X_{m, d}$ and $n:=n_{m, d}$. The Veronese variety can be regarded both as the variety that parameterizes projective classes of homogeneous polynomials of degree $d$ in $m+1$ variables that can be written as $d$-th powers of linear forms, and as the variety that parameterizes projective classes of symmetric tensors $T \in V^{\otimes d}$ where $V$ is a vector space of dimension $m+1$ and $T=v^{\otimes d}$ for certain $v \in V$ (symmetric tensors of the form $v^{\otimes d}$ are often called "completely decomposable tensors"). Hence if we indicate with $\mathbb{K}\left[x_{0}, \ldots, x_{m}\right]_{d}$ the vector space of homogeneous polynomials of degree $d$ in $m+1$ variables, and with $S^{d} V$ the subspace of symmetric tensors in $V^{\otimes d}$, then the Veronese variety $X_{m, d} \subset \mathbb{P}^{n_{m, d}}$ can be described both as $\left\{[F] \in \mathbb{P}\left(K\left[x_{0}, \ldots, x_{m}\right]_{d}\right) \mid \exists L \in\right.$ $K\left[x_{0}, \ldots, x_{m}\right]_{1}$ s.t. $\left.F=L^{d}\right\}$ and as $\left\{[T] \in \mathbb{P}\left(S^{d} V\right) \mid \exists v \in V\right.$ s.t. $\left.T=v^{\otimes d}\right\}$.

A very classical problem coming from a number theory problem known as the Big Waring Problem, consists in determining the minimum integer $s$ for which a generic form $F \in K\left[x_{0}, \ldots, x_{m}\right]_{d}$ can be written as the sum of $s d$-th powers of linear forms $L_{1}, \ldots, L_{s} \in K\left[x_{0}, \ldots, x_{m}\right]_{1}$ :

$$
F=L_{1}^{d}+\cdots+L_{s}^{d}
$$

The same $s$ gives the minimum integer for which the generic symmetric tensor $T \in S^{d} V$ can be written as a sum of $s$ completely decomposable tensors $v_{1}^{\otimes d}, \ldots, v_{s}^{\otimes d} \in S^{d} V$ :

$$
T=v_{1}^{\otimes d}+\cdots+v_{s}^{\otimes d} .
$$

1991 Mathematics Subject Classification. 15A21, 15A69, 14N15.

Key words and phrases. Rank of tensors, Polynomial decomposition, Secant varieties, Veronese Varieties.

The authors were partially supported by CIRM of FBK Trento (Italy), Project Galaad of INRIA Sophia Antipolis Méditerranée (France), Institut Mittag-Leffler (Sweden), Marie Curie: Promoting science (FP7-PEOPLE-2009IEF), MIUR and GNSAGA of INdAM (Italy). 
This problem was solved by J. Alexander and A. Hirschowitz in [2] (see also [5] for a modern proof).

Let $Y \subset \mathbb{P}^{r}$ be any integral and non-degenerate variety. Fix $P \in \mathbb{P}^{r}$. The $Y$-rank $r_{Y}(P)$ of $P$ is the minimal cardinality of a finite set $S \subset Y$ such that $P \in\langle S\rangle$.

Let $\sigma_{s}(Y) \subset \mathbb{P}^{n}$ be the so called " higher $s$-th secant variety of $Y$ " (for brevity we will quote it only as the " $s$-th secant variety of $Y$ "):

$$
\sigma_{s}(Y):=\overline{\bigcup_{P_{1}, \ldots, P_{s} \in Y}\left\langle P_{1}, \ldots, P_{s}\right\rangle} .
$$

From this definition it turns out that a generic element of $\sigma_{s}(Y)$ has $Y$-rank equal to $s$, but obviously not all the elements of $\sigma_{s}(Y)$ have $Y$-rank equal to $s$ (except in some cases, like $s=1$ or $r=\operatorname{dim}(Y)+1)$. For any $P \in \mathbb{P}^{r}$ the $Y$-border $\operatorname{rank} b_{Y}(P)$ of $P$ is the minimal integer $s$ such that $P \in \sigma_{s}(Y)$. We have $b_{Y}(P) \leq r_{Y}(P)$ and equality holds for a general point of each $\sigma_{s}(Y)$. If $Y=X_{m, d}$ and $r=n_{m, d}$, then the integer $r_{X_{m, d}}(P)$ is usually called the symmetric tensor rank or symmetric rank of $P$, while sometimes $b_{X_{m, d}}(P)$ is called either "the secant rank of $P$ " or the "symmetric border rank of $P$ ".

A natural question arising from the applications (see for example [1, [16, 11], 15], 26, 13], [20], 23]) is:

Question 1. Given a symmetric tensor $T \in S^{d} V$ (or a homogeneous polynomial $F \in \mathbb{K}\left[x_{0}, \ldots, x_{m}\right]_{d}$ ), which is the minimum integer $r$ for which we can write it as a linear combination of $r$ completely decomposable tensors, i.e. as in (3) with $r=s$ (or as a linear combination of $r d$-th powers of linear forms, i.e. as in (2) with $r=s$ )?

Obviously answering to Question 1 for $T$ or $F$ is equivalent to finding the $X$-rank of the associated $P \in \mathbb{P}^{n}, n:=n_{m, d}$. Since $\mathbb{P}^{n}$ can be viewed either as the projective space of homogeneous polynomials of degree $d$ in $m+1$ variables or as the projective space of symmetric tensors of order $d$ over an $m+1$ dimensional vector space, an answer to Question 1 for a given $T$ or $F$ (resp. for all $T$ and $F$ ) is equivalent to compute $r_{X}(P)$ for the point $P \in \mathbb{P}^{n}$ associated to $T$ or $F$ (resp. for all $\left.P \in \mathbb{P}^{n}\right)$.

The answer to Question 1] in the particular case of $m=1$, is known since Sylvester ([12, 24], Theorem 4.1, [6], §3). In that case the Veronese variety coincides with a rational normal curve. In [7] and 14] one can find an algebraic theoretical algorithm for the general case with $m \geq 2$.

Both the Big Waring Problem and Question11 have a very interesting reformulation in Algebraic Geometry by using Linear Algebra tools. The authors of [6] give some effective algorithms for the computation of the $X$-rank of certain kind of symmetric tensors by using this algebraic geometric interpretation. The advantage of those last algorithms is that they are effective and that they arise from an algebraic geometric perspective that gives the idea on how one can proceed in the study of the $X$-rank either of a form or of a symmetric tensor. Let us go into the details of that geometric description.

First of all, the definition (44) of the secant varieties of the Veronese variety implies the following chain of containments:

$$
X=\sigma_{1}(X) \subseteq \sigma_{2}(X) \subseteq \cdots \subseteq \sigma_{k-1}(X) \subseteq \sigma_{k}(X)=\mathbb{P}^{n}
$$

for certain natural number $k$. Therefore $\sigma_{s}(X)$ contains all the elements of $X$-rank less or equal than $s$.

Moreover the set

$$
\sigma_{s}^{0}(X):=\bigcup_{P_{1}, \ldots, P_{s} \in X}\left\langle P_{1}, \ldots, P_{s}\right\rangle
$$


is contained in $\sigma_{s}(X)$ and it is made by the elements $P \in \mathbb{P}^{n}$ whose $X$-rank is less or equal than $s$, hence the elements of $\sigma_{s}(X) \backslash\left(\sigma_{s-1}(X) \cup \sigma_{s}^{0}(X)\right)$ have $X$-rank bigger than $s$.

What is done in [6] is to start giving a stratification of $\sigma_{s}(X) \backslash \sigma_{s-1}(X)$ via the $X$-rank: in that paper the cases of $\sigma_{2}\left(X_{m, d}\right)$ and $\sigma_{3}\left(X_{m, d}\right)$ for any $m, d \geq 2$ are completely classified (among others). The authors give algorithms that produce the $X$-rank of an element of $\sigma_{2}\left(X_{m, d}\right)$ and $\sigma_{3}\left(X_{m, d}\right)$.

If we indicate

$$
\sigma_{s, r}(X):=\left\{P \in \sigma_{s}(X) \backslash \sigma_{s-1}(X) \mid r_{X}(P)=r\right\} \subset \sigma_{s}(X) \backslash \sigma_{s-1}(X) \subset \mathbb{P}^{n},
$$

then we can write the stratifications quoted above as follows:

- $\sigma_{2}\left(X_{m, d}\right) \backslash X_{m, d}=\sigma_{2,2}\left(X_{m, d}\right) \cup \sigma_{2, d}\left(X_{m, d}\right)$, for $m \geq 1$ and $d \geq 2$ (cfr. [12, [6], [14, [7]);

- $\sigma_{3}\left(X_{1, d}\right) \backslash \sigma_{2}\left(X_{1, d}\right)=\sigma_{3,3}\left(X_{1, d}\right) \cup \sigma_{3, d-1}\left(X_{1, d}\right)$, for $d \geq 4$ (cfr. [12, 6], [14, [7);

- $\sigma_{3}\left(X_{m, 3}\right) \backslash \sigma_{2}\left(X_{m, 3}\right)=\sigma_{3,3}\left(X_{m, 3}\right) \cup \sigma_{3,4}\left(X_{m, 3}\right) \cup \sigma_{3,5}\left(X_{m, 3}\right)$, for $m \geq 2$ (see [6. Theorem $40])$

- $\sigma_{3}\left(X_{m, d}\right) \backslash \sigma_{2}\left(X_{m, d}\right)=\sigma_{3,3}\left(X_{m, d}\right) \cup \sigma_{3, d-1}\left(X_{m, d}\right) \cup \sigma_{3, d+1}\left(X_{m, d}\right) \cup \sigma_{3,2 d-1}\left(X_{m, d}\right)$, for $m \geq 2$ and $d \geq 4$ (see [6, §4] or [22] for the cases $d=3,4$ ).

What we want to do in this paper is to give the analogous stratification for $\sigma_{4}\left(X_{m, d}\right)$ for any $m, d \geq 2$. We will prove the following:

Theorem 1. The stratification of $\sigma_{4}\left(X_{m, d}\right) \backslash \sigma_{3}\left(X_{m, d}\right)$ via the $X_{m, d}$-rank is the following:

(a) $\sigma_{4}\left(X_{1, d}\right) \backslash \sigma_{3}\left(X_{1, d}\right)=\sigma_{4,4}\left(X_{1, d}\right) \cup \sigma_{4, d-2}\left(X_{1, d}\right)$, if $d \geq 6$;

(b) $\sigma_{4}\left(X_{2,3}\right) \backslash \sigma_{3}\left(X_{2,3}\right)=\mathbb{P}^{9} \backslash \sigma_{3}\left(X_{2,3}\right)=\sigma_{4,4}\left(X_{2,3}\right)$;

(c) $\sigma_{4}\left(X_{2,4}\right) \backslash \sigma_{3}\left(X_{2,4}\right)=\sigma_{4,4}\left(X_{2,4}\right) \cup \sigma_{4,6}\left(X_{2,4}\right) \cup \sigma_{4,7}\left(X_{2,4}\right)$;

(d) $\sigma_{4}\left(X_{2,5}\right) \backslash \sigma_{3}\left(X_{2,5}\right)=\sigma_{4,4}\left(X_{2,5}\right) \cup \sigma_{4,5}\left(X_{2,5}\right) \cup \sigma_{4,7}\left(X_{2,5}\right) \cup \sigma_{4,8}\left(X_{2,5}\right) \cup \sigma_{4,9}\left(X_{2,5}\right)$;

(e) $\sigma_{4}\left(X_{2, d}\right) \backslash \sigma_{3}\left(X_{2, d}\right)=\sigma_{4,4}\left(X_{2, d}\right) \cup \sigma_{4, d-2}\left(X_{2, d}\right) \cup \sigma_{4, d}\left(X_{2, d}\right) \cup \sigma_{4, d+2}\left(X_{2, d}\right) \cup \sigma_{4,2 d-2}\left(X_{2, d}\right)$, if $d \geq 6$;

(f) $\sigma_{4}\left(X_{m, 3}\right) \backslash \sigma_{3}\left(X_{m, 3}\right)=\sigma_{4,4}\left(X_{m, 3}\right) \cup \sigma_{4,5}\left(X_{m, 3}\right) \cup \sigma_{4,6}\left(X_{m, 3}\right) \cup \sigma_{4,7}\left(X_{m, 3}\right)$, if $m \geq 3$;

(g) $\sigma_{4}\left(X_{m, 4}\right) \backslash \sigma_{3}\left(X_{m, 4}\right)=\sigma_{4,4}\left(X_{m, 4}\right) \cup \sigma_{4,6}\left(X_{m, 4}\right) \cup \sigma_{4,8}\left(X_{m, 4}\right) \cup \sigma_{4,10}\left(X_{m, 4}\right)$, if $m \geq 3$;

(h) $\sigma_{4}\left(X_{m, 5}\right) \backslash \sigma_{3}\left(X_{m, 5}\right)=\sigma_{4,4}\left(X_{m, 5}\right) \cup \sigma_{4,5}\left(X_{m, 5}\right) \cup \sigma_{4,7}\left(X_{m, 5}\right) \cup \sigma_{4,8}\left(X_{m, 5}\right) \cup \sigma_{4,10}\left(X_{m, 5}\right) \cup$ $\sigma_{4,13}\left(X_{m, 5}\right)$, if $m \geq 3$;

(i) $\sigma_{4}\left(X_{m, d}\right) \backslash \sigma_{3}\left(X_{m, d}\right)=\sigma_{4,4}\left(X_{m, d}\right) \cup \sigma_{4, d-2}\left(X_{m, d}\right) \cup \sigma_{4, d}\left(X_{m, d}\right) \cup \sigma_{4, d+2}\left(X_{m, d}\right) \cup \sigma_{4,2 d-2}\left(X_{m, d}\right) \cup$ $\sigma_{4,2 d}\left(X_{m, d}\right) \cup \sigma_{4,3 d-2}\left(X_{m, d}\right)$, if $m \geq 3$ and $d \geq 6$.

Moreover all listed $\sigma_{s, r}\left(X_{n, d}\right)$ are non-empty.

The case of the rational normal curve, i.e. item (a), is due to Sylvester and done in [12, 14, 7], 24, Theorem 4.1], and [6, §3].

The cases of the Veronese surfaces in degrees 3 and 4, i.e. items (b) and (c), are done in 6 . Theorems 40, 44] respectively and in 22].

We complete the case of the Veronese surfaces (items (d) and (e)) in the Subsection 1 In the Subsection 4.2 we will give the stratification of $\sigma_{4}\left(X_{3, d}\right)$, with $d \geq 3$, that will be the same stratification for any $m \geq 3$ (items (f), (g), (h) and (i)).

Remark 1. During the Spring Semester 2001 "Algebraic Geometry with a view towards applications" at the Institut Mittag-Leffler (Sweeden), J. H. Rhodes stated the following:

Conjecture 1 (J. H. Rhodes): The maximal $X_{m, 3}$-rank of a homogeneous polynomial of degree 3 in $m+1$ variables belonging to $\sigma_{m+1}\left(X_{m, 3}\right)$ is $2(m+1)-1$.

We remark that the original version of this conjecture was about tensors not necessarily symmetric. For sake of completeness we quote the first version of it but we don't enter into details.

Conjecture 0 (J. H. Rhodes): The maximal rank of an $(m+1) \times(m+1) \times(m+1)$ tensor of border rank $m+1$ is $2(m+1)-1$. 
Conjecture 1 is known to be true for $m=1,2(m=1$ is classical from Sylvester, and $m=2$ is $\underline{6}$, Theorem 40]). We like to observe here that the case (f) of our Theorem 1 shows that this conjecture is true also for $m=3$. Moreover from the proof of our Theorem 1, it is also possible to describe the structure of the schemes that evince the $X_{m, 3}$-border rank of the degree 3 polynomials of maximal $X_{m, 3}$-rank (they are all described by Proposition 10). Finally, putting $d=3$ and $m=3$, one can use our Remark 9 to produce algorithmically several sets of points evincing $r_{X_{3,3}}(P)$ for points $P \in \sigma_{4}\left(X_{3,3}\right)$ of maximal $X_{3,3}$-rank.

Before going into the details of the proof we need some preliminary and auxiliary sections. In Section 1 we present the construction that will allow to associate two different 0-dimensional schemes of $\mathbb{P}^{m}$ to two 0-dimensional sub-schemes of $X_{m, d}$ realizing the $X_{m, d}$-border rank and the $X_{m, d}$-rank of a point $P \in \mathbb{P}^{n_{m, d}}$. We also discuss for which 0 -dimensional schemes $A \subset \mathbb{P}^{m}$ of degree 4 a general point of $\left\langle\nu_{m, d}(A)\right\rangle$ belongs to $\sigma_{4}\left(X_{m, d}\right) \backslash \sigma_{3}\left(X_{m, d}\right)$. In Section 2 we give bounds for the $Y$-rank of a point with respect to some particular projective curves $Y \subset \mathbb{P}^{t}$ that will be used in the proof of the Theorem 1 Section 3 is made by preliminary lemmas on the linear dependence of the pre-image via the Veronese map $\nu_{m, d}$ of the 0 -dimensional schemes realizing the $X$-rank and the $X$-border rank of a point $P \in \mathbb{P}^{n}$. Finally in Section 4 we collect all the previous results into the proof of Theorem 1 .

Moreover we will describe case by case how to find the scheme that realizes the $X$-rank of a point $P$ (modulo the scheme that realizes the $X$-border rank). This allows us to give many informations on the subset $\sigma_{s, r}(X) \subset \sigma_{s}(X)$ defined in (7) and to construct all $P \in \sigma_{4, s}(X)$ by playing with certain 0 -dimensional schemes.

We like to stress here that the defining ideals of $\sigma_{2}\left(X_{1, d}\right)$ and $\sigma_{3}\left(X_{2, d}\right)$ are known (see [21] and 27 respectively) and this allows the authors of 6 to give algorithms for the $X$-rank of points in $\sigma_{s}(X)$ with $s=2,3$. Given an element $P \in \mathbb{P}^{n}$ they can firstly check if its $X$-border rank is actually either 2 or 3 , and then they can produce the algorithm for the computation of the $X$-rank of $P$. Unfortunately, at our knowledge, equations defining $\sigma_{4}\left(X_{3, d}\right)$ at least set-theoretically are not known yet, therefore we could write algorithms for the $X$-rank of an element $P \in \sigma_{4}(X)$ only if we already knew in some way that $b_{X}(P)=4$.

\section{Preliminaries}

In this paper we want to study the $X$-rank of the points $P$ belonging to the fourth secant variety of the Veronese variety $X$, i.e. $P \in \sigma_{4}(X)$. By the chain of containments (5) we have that $\sigma_{3}(X) \subseteq \sigma_{4}(X)$. Since the stratification of $\sigma_{3}(X)$ via the $X$-rank is already known by $[6$, it is sufficient to understand the $X$-rank of points $P \in \sigma_{4}(X) \backslash \sigma_{3}(X)$.

Moreover the definition (6) of $\sigma_{s}^{0}(X)$ implies that if $P \in \sigma_{4}^{0}(X)$ then $r_{X}(P) \leq 4$, hence, for the purpose of this paper, it is sufficient to study the $X$-rank of points belonging to $\sigma_{4}(X) \backslash\left(\sigma_{3}(X) \cup\right.$ $\left.\sigma_{4}^{0}(X)\right)$. Before starting our construction by taking $P \in \sigma_{4}(X)$ we introduce the following Remark 2 that, for such a point $P$, gives the existence of a 0 -dimensional scheme $Z \subset X$ of degree 4 such that $P \in\langle Z\rangle$ and $P \notin\left\langle Z^{\prime}\right\rangle$ for any $Z^{\prime} \subset X$ with $\operatorname{deg}\left(Z^{\prime}\right)<\operatorname{deg}(Z)$.

Remark 2. Fix integers $m \geq 1, d \geq 2$ and $P \in \mathbb{P}^{n}$ such that $b_{X}(P) \leq d+1$. By [9], Lemma 2.1.5 and Lemma 2.4.4, there is a smoothable 0 -dimensional and Gorenstein scheme $\mathcal{E} \subset X_{m, d}$ such that $\operatorname{deg}(\mathcal{E})=b_{X}(P), P \in\langle\mathcal{E}\rangle$ and $P \notin\left\langle\mathcal{E}^{\prime}\right\rangle$ for any $\mathcal{E}^{\prime} \subsetneq \mathcal{E}$.

Before entering into the details of our construction we need to distinguish the Gorenstein cases from the non Gorenstein ones that we won't have to treat thanks to 9 .

Lemma 1. Let $U$ be smooth quasi-projective surface and fix $O \in U$. Let $2 O$ be the 0 -dimensional subscheme of $U$ with $\left(\mathcal{I}_{O, U}\right)^{2}$ as its ideal sheaf. Let $E \subset U$ be another 0-dimensional scheme 
supported at $O$ with $\operatorname{deg}(E)=4$. Assume that $E$ is Gorenstein, but not curvilinear. The latter condition is equivalent to requiring $2 O \subseteq E$. Let $\mathbb{K}[[x, y]]$ be the completion of $\mathcal{O}_{U, O}$ and let $J \subset \mathbb{K}[[x, y]]$ be an ideal such that $\mathcal{O}_{E} \cong \mathbb{K}[[x, y]] / J$. Then $J$ is generated by $L_{1}^{2}$ and $L_{2}^{2}$ with $L_{1}, L_{2} \in \mathbb{K}[x, y]_{1}$.

Proof. Since $\operatorname{dim}(U)=2, U$ is smooth and $E_{\text {red }}=\{O\}, E$ is not curvilinear if and only if $2 O \subseteq E$. Since $\mathbb{K}[[x, y]]$ is a regular local ring and $J$ is a codimension 2 Gorenstein ideal, $J$ is a complete intersection ([17, Corollary 21.20]). Take generators $f_{1}, f_{2}$ of $J$ and call $Q_{i}(x, y)$ the first nonzero term of the Taylor expansion of $f_{i}$. Set $\mu_{i}:=\operatorname{deg}\left(Q_{i}(x, y)\right)$. Since $E$ is not curvilinear, we have $\mu_{i} \geq 2$ for all $i$. Since $\operatorname{deg}(E)=4=2 \cdot 2$, we have $\mu_{1}=\mu_{2}=2$ and $Q_{1}, Q_{2}$ have no common factor (Property (5) of [19, §3.3]). See $x, y$ as homogeneous coordinates of $\mathbb{P}^{1}$. The pencil $\lambda Q_{1}+\mu Q_{2},(\lambda, \mu) \in \mathbb{K}^{2} \backslash\{(0,0)\}$, has at least one singular element. Hence, up to a linear change of coordinates, we may assume $Q_{1}=x^{2}$ and write $Q_{2}=a x y+b y^{2}$. Since $Q_{1}$ and $Q_{2}$ have no common factor, we have $b \neq 0$. Set $L_{1}:=x$ and $L_{2}:=y+a x / 2 b$. Since $\left(Q_{1}, Q_{2}\right)=\left(L_{1}^{2}, L_{2}^{2}\right)=$ $\left(L_{1}^{2}, L_{2}^{2}, x^{3}, x^{2} y, x y^{2}, y^{3}\right)=\left(Q_{1}, Q_{2}, x^{3}, x^{2} y, x y^{2}, y^{3}\right) \supseteq J$ and $\mathbb{K}[[x, y]] /\left(L_{1}^{2}, L_{2}^{2}\right)$ is a 4-dimensional $\mathbb{K}$-vector space, we get $J=\left(L_{1}^{2}, L_{2}^{2}\right)$.

Remark 3. As already observed, thanks to [9, Lemma 2.4.4], in the list of all cases potentially appearing in Theorem 1 we will only need to check the Gorenstein schemes $A \subset \mathbb{P}^{m}$ with $\operatorname{deg}(A)=$ 4. The scheme $A$ is Gorenstein if and only if every connected component of $A$ is Gorenstein. Let $E$ be a connected 0-dimensional scheme of degree $s \leq 4$. If $s \leq 3$, then it is Gorenstein if and only if it is curvilinear and it is one of the ones appearing in [6, §4] in the description of $\sigma_{s}(X)$. Now assume $s=4$. Let $e$ be the embedding dimension of $E$, i.e. set $e:=\operatorname{dim}_{\mathbb{K}}\left(\mathbf{m}_{E} / \mathbf{m}_{E}^{2}\right)$, where $\mathbf{m}_{E}$ is the maximal ideal of the local ring $\mathcal{O}_{E}$. Since $\mathcal{O}_{E} / \mathbf{m}_{E}=\mathbb{K}$, we have $e \leq \operatorname{deg}(E)-1=3$ and equality holds if and only if $\mathbf{m}_{E}^{2}=0$, i.e. if and only if $E$ is isomorphic to the scheme $\mathbb{K}[x, y, z] /(x, y, z)^{2}$. Since the latter scheme is not Gorenstein, it is sufficient to look at the case $e \leq 2$. If $e=1$, then $E$ is Gorenstein, because every effective divisor of a smooth curve is a locally complete intersection and any local complete intersection is Gorenstein ([17, Corollary 21.29]). The case $e=2$ is described in Lemma 1 .

If $d \geq 7$ the scheme evincing the border rank is unique, because the union of two such schemes has degree at most 8 and we may apply [4 Lemma 1] (stated in Section 2 as Lemma 2) to these schemes instead of $A$ and $B$. Hence each $P \in \sigma_{4}(X)$ is associated to a unique $Z$ and $\left\langle\nu_{m, d}\left(A_{1}\right)\right\rangle \cap\left\langle\nu_{m, d}\left(A_{2}\right)\right\rangle=\left\langle\nu_{m, d}\left(A_{1} \cap A_{2}\right)\right\rangle$ for all $A_{i} \subset \mathbb{P}^{m}$ with $\operatorname{deg}\left(A_{i}\right) \leq 4, i=1,2$. Only if $d \geq 7$ we claim that a general $P \in\langle Z\rangle$ belongs $\sigma_{4}(X) \backslash \sigma_{3}(X)$.

Definition 1. Let $P \in \sigma_{4}(X) \backslash \sigma_{3}(X)$; we say that a 0 -dimensional scheme $Z \subset X$ such that $\operatorname{deg}(Z)=4$ and $P \in\langle Z\rangle$ " evinces the $X$-border rank of $P$ ".

Notation 1. If $P \in \sigma_{4}(X) \backslash\left(\sigma_{3}(X) \cup \sigma_{4}^{0}(X)\right)$, we fix $Z \subset X$ to be one of the degree 4 non-reduced 0-dimensional schemes that evince the $X$-border rank of $P$, i.e. $P \in\langle Z\rangle$ and $P \notin\left\langle Z^{\prime}\right\rangle$ for all 0-dimensional schemes $Z^{\prime} \subset X$ of degree less or equal than 3. If $Z=\nu_{m, d}(A)$, then we also say (with an abuse of notation) that $A$ evinces the $X_{m, d}$-border rank of $P$.

In order to study the stratification of $\sigma_{4}\left(X_{m, d}\right)$ it is therefore necessary to understand the $X_{m, d^{-}}$ rank of the points belonging to the span of a non reduced 0-dimensional subscheme $Z \subset X_{m, d}$ of degree 4. Clearly, for such a degree 4 scheme $Z$ we have that $\operatorname{dim}(\langle Z\rangle) \leq 3$. By [25. Proposition 3.1], or [24, Subsection 3.2], [6, Remark 31], it is sufficient to do the cases $m=2,3$. The stratification of $\sigma_{4}\left(X_{1, d}\right)$ is already known by [12] and [6]. Hence it remains to study the stratification of $\sigma_{4}\left(X_{2, d}\right)$ for $d \geq 5$ (in fact [6] and [22] give it for the cases $d=3,4$ ), and the stratification of $\sigma_{4}\left(X_{m, d}\right)$ for $m \geq 3$. What the already quoted results in [6, 24] and 25] allow to do is that, once we will have the stratification of $\sigma_{4}\left(X_{3, d}\right)$ then we will straightforwardly have that the same stratification will hold for $\sigma_{4}\left(X_{m, d}\right)$ for the same $d$ and $m \geq 3$. 
By the discussion above we may assume that the scheme $A$ defined in Notation 1 is not contained in a 2-dimensional projective subspace of $\mathbb{P}^{m}$. In fact, if $\operatorname{deg}(A)=4$ then $\langle A\rangle \subseteq \mathbb{P}^{3}$, but if $\langle A\rangle \subseteq \mathbb{P}^{2}$ then there exist a 0 -dimensional scheme $B \subset \mathbb{P}^{2}$ of degree 3 such that $\langle A\rangle \subseteq\langle B\rangle=\mathbb{P}^{2}$. This would imply that any point $P \in\left\langle\nu_{m, d}(A)\right\rangle$ belongs to $\left\langle\nu_{m, d}(B)\right\rangle$ for some 0-dimensional scheme $B \subset \mathbb{P}^{m}$ of degree 3. Now, since $\operatorname{deg}(B)=3$ then $\left\langle\nu_{m, d}(B)\right\rangle \subset \sigma_{3}(X)$. Therefore if $\langle A\rangle \subset \mathbb{P}^{2}$ we get that, if $Z=\nu_{m, d}(A)$, any point $P \in\langle Z\rangle$ belongs to $\sigma_{3}(X)$, but we want to study the $X$-rank of the points $P \in \sigma_{4}(X) \backslash \sigma_{3}(X)$. Therefore we assume that the scheme $A \subset \mathbb{P}^{m}$ defined in Notation 1 spans a projective subspace of dimension 3 .

Notation 2. Let $P \in \sigma_{4}\left(X_{m, d}\right) \backslash\left(\sigma_{3}\left(X_{m, d}\right) \cup \sigma_{4}^{0}\left(X_{m, d}\right)\right)$. We fix a finite set $S \subset X_{m, d}$ that evinces the $X_{m, d}$-rank of $P$, i.e. $S \subset X_{m, d}$ is a finite set such that $P \in\langle S\rangle$ and $\sharp(S)=r_{X_{m, d}}(P)$. Let $B \subset \mathbb{P}^{m}$ be the only set such that $\nu_{d, m}(B)=S$. We also say that $B$ evinces the $X_{m, d}$-rank of $P$.

This notation allows us to use many results on the reduced and non-reduced 0-dimensional schemes in $\mathbb{P}^{m}$ and translate them into informations on the 0-dimensional sub-schemes of $X_{m, d}$.

\section{UsEFul REDUCIBLE CURVES}

Let $Y$ be a projective non-degenerate reduced curve obtained by the union of two rational normal curves $Y_{1}, Y_{2}$. We prove here two propositions on the $Y$-rank of points belonging to $\langle Z\rangle$ where $Z$ is a degree 4 non-reduced 0 -dimensional sub-scheme of $Y$.

We'd like to point out that in Proposition 1 we will only give an upper bound for the $Y$-rank of certain points, but in Section 4 it will be proved that this upper bound is the actual value of the $Y$-rank (Corollary 2).

Proposition 1. Fix an integer $d \geq 3$. Let $Y \subset \mathbb{P}^{2 d}$ be a reduced and connected curve union of two smooth degree $d$ curves $Y_{1}, Y_{2}$, each of them a rational normal curve in its linear span, with a unique common point, $Q$, and with $\langle Y\rangle=\mathbb{P}^{2 d}$. Let $Z \subset Y$ be a 0-dimensional scheme such that $\operatorname{deg}(Z)=4, Z_{\text {red }}=\{Q\}, Z$ is a Cartier divisor of $Y$ and $\operatorname{deg}\left(Z \cap Y_{i}\right) \geq 2$ for $i=1,2$. Fix $P \in\langle Z\rangle$ such that $P \notin\left\langle Z^{\prime}\right\rangle$ for any $Z^{\prime} \subsetneq Z$. Then:

$$
r_{Y}(P) \leq 2 d-2
$$

and there is a reduced 0-dimensional sub-scheme $S \subset Y$ such that $P \in\langle S\rangle, Q \notin S, \sharp(S)=2 d-2$, and $\sharp\left(S \cap Y_{i}\right)=d-1$ for $i=1,2$. We may find $S$ as above and not intersecting any finite prescribed subset of $Y$.

If $d \geq 4$, then for a general pair of sets of points $\left(A_{1}, A_{2}\right) \subset Y_{1} \times Y_{2}$ such that $\sharp\left(A_{1}\right)=\sharp\left(A_{2}\right)=d-3$ there is $S$ as above with the additional property that $A_{1} \cup A_{2} \subset S$.

Proof. (a) First assume $d=3$. Let $\ell_{P}: \mathbb{P}^{6} \backslash\{P\} \rightarrow \mathbb{P}^{5}$ be the linear projection from $P$. Since $P \notin Y$, then the map $\ell_{P} \mid Y$ is obviously a morphism. Since $P \notin\left\langle Y_{i}\right\rangle$, each curve $C_{i}:=\ell_{P}\left(Y_{i}\right)$, $i=1,2$, is a rational normal curve in its 3 -dimensional linear span. Since $\left\langle Y_{1}\right\rangle \cap\left\langle Y_{2}\right\rangle=\{Q\}$, the linear space $D:=\left\langle C_{1}\right\rangle \cap\left\langle C_{2}\right\rangle$ has dimension at most 1 . Since $\operatorname{dim}\left(\left\langle C_{i}\right\rangle\right)=3$ for all $i$, we have $\operatorname{dim}(D)=1$. Now the 0 -dimensional sub-scheme $Z \subset Y$ is, by hypothesis, such that $P \in\langle Z\rangle$ and $P \notin\left\langle Z^{\prime}\right\rangle$ for any proper sub-scheme $Z^{\prime} \subset Z$. If $P \in\left\langle T_{Q} Y_{1} \cup T_{Q} Y_{2}\right\rangle$ we would have that $P$ belongs to the span of a proper sub-scheme of $Z$ of degree 3 (in fact $\operatorname{dim}\left(\left\langle T_{Q} Y_{1} \cup T_{Q} Y_{2}\right\rangle\right.$ ) $=2$, because $Q \in Y_{1} \cap Y_{2}$, while $\operatorname{dim}(\langle Z\rangle)=3$ because $\left.d \geq 3\right)$ that contradicts the hypothesis. Since $P \in\langle Z\rangle \backslash\left\langle T_{Q} Y_{1} \cup T_{Q} Y_{2}\right\rangle$, the line $D$ is not tangent either to $C_{1}$ or to $C_{2}$, but it intersects each $C_{i}$ only at their common point $\ell_{P}(Q)$. Hence the linear projection from $D$ induces a degree 2 morphism $\psi_{i}: C_{i} \rightarrow \mathbb{P}^{1}$. Thus, for a general $O \in D$, there are two sets of points $B_{i} \subset C_{i}$ such that $\sharp\left(B_{i}\right)=2$ and $O \in\left\langle B_{i}\right\rangle$, for $i=1,2$. Let $S_{i} \subset Y_{i}$ be the only set of points such that $\ell_{P}\left(S_{i}\right)=B_{i}$ for $i=1,2$. Since $\operatorname{dim}\left(\left\langle\ell_{P}\left(S_{1} \cup S_{2}\right)\right\rangle\right)=2$, we have $\operatorname{dim}\left(\left\langle\{P\} \cup S_{1} \cup S_{2}\right\rangle\right)=3$. We easily find 
$O \in D$ such that $\operatorname{dim}\left(\left\langle S_{1} \cup S_{2}\right\rangle\right)=3$ and $Q \notin S_{1} \cup S_{2}$. Hence $P \in\left\langle S_{1} \cup S_{2}\right\rangle$. We can then take $S:=S_{1} \cup S_{2}$ as a solution for $d=3$.

(b) Now assume $d \geq 4$. Take a general pair of sets of points $\left(A_{1}, A_{2}\right) \subset Y_{1} \times Y_{2}$ such that $\sharp\left(A_{1}\right)=\sharp\left(A_{2}\right)=d-3$. Let $\ell: \mathbb{P}^{2 d} \backslash\left\langle A_{1} \cup A_{2}\right\rangle \rightarrow \mathbb{P}^{6}$ denote the linear projection from $\left\langle A_{1} \cup A_{2}\right\rangle$. Apply Step (a), i.e. the case $d=3$, to the curve $Y^{\prime} \subset \mathbb{P}^{6}$ which is the closure of $\ell\left(Y \backslash Y \cap\left\langle A_{1} \cup A_{2}\right\rangle\right)$. Let $S_{1} \cup S_{2}$ be a solution for $Y^{\prime}$ with respect to the point $\ell(P)$. For general $O \in D$ (as in Step (a)) we may find $S_{1} \cup S_{2}$ not through the finitely many points of $Y^{\prime}$ which are in $Y^{\prime} \backslash \ell\left(Y \backslash\left(A_{1} \cup A_{2}\right)\right)$. Hence there are unique $B_{i} \subset Y_{i}$ such that $\ell\left(B_{i}\right)=S_{i}$ for $i=1$, 2. Set $S:=B_{1} \cup B_{2} \cup A_{1} \cup A_{2}$.

In Corollary 2 we will show that (8) is an equality.

Proposition 2. Fix an integer $d \geq 3$. Let $Y \subset \mathbb{P}^{2 d+1}$ be a reduced curve union of two smooth degree $d$ curves $Y_{1}, Y_{2}$, each of them a rational normal curve in its linear span and such that $\left\langle Y_{1}\right\rangle \cap\left\langle Y_{2}\right\rangle=\emptyset$. Fix $P_{i} \in Y_{i}$ for $i=1,2$. Let $Z_{i} \subset Y_{i}$ be the degree 2 effective Cartier divisor $2 P_{i}$ of $Y_{i}, i=1,2$. Set $Z:=Z_{1} \cup Z_{2}$. Fix $P \in\langle Z\rangle$ such that $P \notin\left\langle Z^{\prime}\right\rangle$ for all $Z^{\prime} \subsetneq Z$. Then $b_{Y}(P)=4$, $Z$ is the only subscheme of $Y$ evincing $b_{Y}(P)$,

$$
r_{Y}(P)=2 d,
$$

and $\sharp\left(S \cap Y_{1}\right)=\sharp\left(S \cap Y_{2}\right)=d$ for all reduced 0-dimensional sub-schemes $S \subset Y$ evincing $r_{Y}(P)$.

Proof. Since $\operatorname{deg}(Z)=4$ and since $Y$ is a smooth curve, we have $b_{Y}(P) \leq 4$.

Obviously $\langle Z\rangle \cap\left\langle Y_{i}\right\rangle \supseteq\left\langle Z_{i}\right\rangle$. Let's see the other containment. We show that $\langle Z\rangle \cap\left\langle Y_{1}\right\rangle \subseteq\left\langle Z_{1}\right\rangle$ (the same proof holds for $\langle Z\rangle \cap\left\langle Y_{2}\right\rangle \subseteq\left\langle Z_{2}\right\rangle$ ). If $\langle Z\rangle \cap\left\langle Y_{1}\right\rangle$ is not contained in $\left\langle Z_{1}\right\rangle$, then there exists a point $Q \in\langle Z\rangle \cap\left\langle Y_{1}\right\rangle$ such that $Q \notin\left\langle Z_{1}\right\rangle$. Therefore $\left\langle Z_{1}, Q\right\rangle$ is a plane $\Pi$, and since $Q \in\langle Z\rangle$, we have that $\left\langle Z_{1}, Q\right\rangle:=\Pi \subset\langle Z\rangle$. Now, by construction, $Q \in\left\langle Y_{1}\right\rangle$, hence $\Pi$ is spanned by a 0 -dimensional scheme of degree 3 that is contained in $\left\langle Y_{1}\right\rangle$, by hypothesis $\left\langle Y_{1}\right\rangle \cap\left\langle Y_{2}\right\rangle=\emptyset$, then $\Pi$ cannot intersect $\left\langle Z_{2}\right\rangle$ which is entirely contained in $\left\langle Y_{2}\right\rangle$. Both $\Pi$ and $\left\langle Z_{2}\right\rangle$ are contained in $\langle Z\rangle$ which has projective dimension 3 . Therefore, if such a $Q$ exists, we would have a projective plane $\Pi$ and a line $\left\langle Z_{2}\right\rangle$ that are contained in a $\mathbb{P}^{3}$ without intersecting each other, but this is impossible. Then $\langle Z\rangle \cap\left\langle Y_{i}\right\rangle \subseteq\left\langle Z_{i}\right\rangle$ for $i=1,2$.

Since $P \notin\left\langle Z^{\prime}\right\rangle$ for all $Z^{\prime} \subsetneq Z$, we get $P \notin\left\langle Y_{1}\right\rangle$ and $P \notin\left\langle Y_{2}\right\rangle$. Since for $i=1,2 Y_{i}$ is a rational normal curve, then $Z_{i}$ is the only sub-scheme of $Y_{i}$ evincing $b_{Y_{i}}(Q)$ for all $Q \in T_{P_{i}} Y_{i} \backslash\left\{P_{i}\right\}$. We immediately get that $Z$ is the only sub-scheme of $Y$ with length at most 4 whose linear span contains $P$. Hence we have proved that $Z$ is the unique 0 -dimensional scheme that evinces the $Y$-border rank of $P$ and that $P \in \sigma_{4}(Y) \backslash \sigma_{3}(Y)$.

Now we compute $r_{Y}(P)$.

Let $\ell_{P}: \mathbb{P}^{2 d+1} \backslash\{P\} \rightarrow \mathbb{P}^{2 d}$ denote the linear projection from $P$. Set $C:=\ell_{P}(Y)$ and $C_{i}:=\ell_{P}\left(Y_{i}\right)$. Since $P \notin \sigma_{2}(Y)$, then $\ell_{P} \mid Y$ is an embedding. Hence $C_{1} \cap C_{2}=\emptyset$. Since $P \notin\left\langle Y_{1}\right\rangle \cup\left\langle Y_{2}\right\rangle$, then each $C_{i}$ is a degree $d$ rational normal curve in its linear span. Thus $r_{Y}(P)$ is the minimal cardinality of a set of points $A:=A_{1} \cup A_{2}$ such that $A_{1} \subset C_{1}, A_{2} \subset C_{2}$ and $A_{1} \cup A_{2}$ is linearly dependent. Notice that $\left\langle C_{1}\right\rangle \cap\left\langle C_{2}\right\rangle$ is a unique point $O \notin C$. Set $Q_{i}:=\ell_{P}\left(P_{i}\right)$ with $P_{i}=\left(Z_{i}\right)_{\text {red }}, W_{i}:=\ell_{P}\left(Z_{i}\right)$ and $W:=W_{1} \cup W_{2}$. Hence $W_{i}$ is the degree 2 effective divisor $2 Q_{i}$ of $C_{i}$. Since $P \in\langle Z\rangle$, then $\langle W\rangle$ is a plane. Thus the two lines $T_{Q_{i}} C_{i}, i=1,2$, meet each other. Since $\{O\}=\left\langle C_{1}\right\rangle \cap\left\langle C_{2}\right\rangle$, then $O$ is their unique common point. Since $O \in T_{Q_{i}} C_{i}$, we have $r_{C_{i}}(O)=d$ (see [12] or [24, Theorem 4.1]). Hence $\sharp\left(A \cap C_{1}\right) \geq d$ and $\sharp\left(A \cap C_{2}\right) \geq d$. Since $\left\langle C_{1}\right\rangle \cap\left\langle C_{2}\right\rangle=\{O\}$ and any $d+1$ points of $C_{i}$ are linearly independent, $\sharp\left(A \cap C_{1}\right)=d$ and $\sharp\left(A \cap C_{2}\right)=d$ for every linearly dependent $A \subset C$ such that $\sharp\left(A \cap C_{i}\right) \leq d$ for all $i$. Then $r_{Y}(P) \geq 2 d$, but $r_{Y_{i}}\left(R_{i}\right)=d$ for all $R_{i} \in T_{P_{i}} Y_{i} \backslash Y_{i}$ and $i=1,2$, hence $r_{Y}(P) \leq 2 d$ and therefore $r_{Y}(P)=2 d$. 


\section{LEMmas}

In Notation 2 and in Notation 1 we defined two different 0-dimensional schemes $Z, S \subset X$ realizing the $X$-border rank and the $X$-rank respectively of a point $P \in \sigma_{4}(X) \backslash\left(\sigma_{4}^{0}(X) \cup \sigma_{3}(X)\right)$ and two 0-dimensional schemes $A, B \subset \mathbb{P}^{m}$ such that $\nu_{m, d}(A)=Z$ and $\nu_{m, d}(B)=S$ respectively. Here, but only for this Section 3, we do not care about the fact that $P \in\left\langle\nu_{m, d}(A)\right\rangle \cap\left\langle\nu_{m, d}(B)\right\rangle$ is a point of $\sigma_{4}(X) \backslash\left(\sigma_{4}^{0}(X) \cup \sigma_{3}(X)\right)$ : for this section $A, B \subset \mathbb{P}^{m}$ are 0 -dimensional schemes whose images via $\nu_{m, d}$ still realize the $X$-border rank and the $X$-rank respectively of a point $P \in \mathbb{P}^{n}$, but here we do not give any restriction on the minimum secant variety $\sigma_{s}(X)$ such that $P \in \sigma_{s}(X)$. This is summarized in the following notation.

Notation 3. In this section, and only in this section, when considering a point $P \in \mathbb{P}^{n}$, we assume that:

- $A \subset \mathbb{P}^{m}$ is a non-reduced 0-dimensional scheme such that $\nu_{m, d}(A)=Z \subset \mathbb{P}^{n}$ evinces the $X$-border rank of $P \in \mathbb{P}^{n}$,

- $B \subset \mathbb{P}^{m}$ is a reduced 0-dimensional scheme such that $\nu_{m, d}(B)=S \subset \mathbb{P}^{n}$ evinces the $X$-rank of $P \in \mathbb{P}^{n}$,

- $\operatorname{deg}(A)<\operatorname{deg}(B)$.

More assumptions on the degrees of $A$ and $B$ will be explained in each Lemma.

We recall the following result ([4, Lemma 1]).

Lemma 2. Fix $P \in \mathbb{P}^{n_{m, d}}$. Let $A, B \in \mathbb{P}^{m}$ be two 0-dimensional schemes as in Notation 3 , Then $h^{1}\left(\mathbb{P}^{m}, \mathcal{I}_{A \cup B}(d)\right)>0$.

We introduce here a tool that we will use in the proofs of the next lemmata.

Notation 4. Let $E \subset \mathbb{P}^{m}$ be a 0-dimensional scheme and let $H \subset \mathbb{P}^{m}$ be a hyperplane, then the sequence that defines the residual scheme $\operatorname{Res}_{H}(E)$ of $E$ with respect to $H$ is the following:

$$
0 \rightarrow \mathcal{I}_{\operatorname{ReS}_{H}(E)}(t-1) \rightarrow \mathcal{I}_{E}(t) \rightarrow \mathcal{I}_{E \cap H, H}(t) \rightarrow 0 .
$$

Lemma 3. Fix an integer $d \geq 2$ and a 0-dimensional and curvilinear subscheme $E$ of $\mathbb{P}^{2}$ such that $\operatorname{deg}(E)=2 d+2$ and $h^{1}\left(\mathcal{I}_{E}(d)\right)>0$. Assume that $E$ has at least one reduced component.

(i) If $E$ is in linearly general position, then $h^{1}\left(\mathcal{I}_{E}(d)\right)=1$ and there is a smooth conic $C$ such that $E \subset C$.

(ii) If $E$ is not in linearly general position, then either there is a line $L \subset \mathbb{P}^{2}$ such that $\operatorname{deg}(L \cap E) \geq d+2$ and $h^{1}\left(\mathcal{I}_{E}(d)\right)=\operatorname{deg}(L \cap E)-d-1$ or there is a singular conic $\Gamma$ such that $E \subset \Gamma$. The fact that $E$ has at least one reduced component, implies that $\Gamma$ is reduced, say $\Gamma=L_{1} \cup L_{2}$, with $L_{1} \neq L_{2}, \sharp\left(E \cap L_{1}\right)=\sharp\left(E \cap L_{2}\right)=d+1$ and $E$ is a Cartier divisor of $L_{1} \cup L_{2}$.

Proof. First assume that $E$ is in linearly general position. Let $C \subset \mathbb{P}^{2}$ be a conic such that $y:=\operatorname{deg}(E \cap C)$ is maximal. Since $\operatorname{deg}(E) \geq 5$ and $\left(\begin{array}{l}4 \\ 2\end{array}\right)=6$, we have $y \geq 5$. Since no line contains at least 3 points of $E$, the conic $C$ is smooth. Since $\operatorname{Res}_{C}(E) \subset E$, the scheme $\operatorname{Res}_{C}(E)$ is in linearly general position. Since $\operatorname{deg}\left(\operatorname{Res}_{C}(E)\right)=2 d+2-y \leq 2(d-2)+1$, we have $h^{1}\left(\mathcal{I}_{\operatorname{Res}_{C}(E)}(d-2)\right)=0$ ([18, Theorem 3.2]). Thus the exact sequence

$$
0 \rightarrow \mathcal{I}_{\operatorname{Res}_{C}(E)}(d-2) \rightarrow \mathcal{I}_{E}(d) \rightarrow \mathcal{I}_{C \cap E}(d) \rightarrow 0
$$

gives $h^{1}\left(C, \mathcal{I}_{C \cap E}(d)\right)>0$. Thus $\operatorname{deg}(E \cap C) \geq 2 d+2$. Since $\operatorname{deg}(E)=2 d+2$, we get $E \subset C$, concluding the proof of (i).

Now assume that $E$ is not in linearly general position. Take a line $L \subset \mathbb{P}^{2}$ such that $x:=$ $\operatorname{deg}(L \cap E)$ is maximal. By assumption we have $x \geq 3$. First assume $x \geq d+2$. Since $\operatorname{Res}_{L}(E)$ has degree $2 d+2-x$, we have $h^{1}\left(\mathcal{I}_{\operatorname{Res}_{L}(E)}(d-1)\right)=0([6$, Lemma 34]). From the exact sequence (9) 
we get the result in this case. Now assume $x \leq d+1$. If $h^{1}\left(\mathcal{I}_{\operatorname{Res}_{L}(E)}(d-1)\right)=0$, then (9) gives $h^{1}\left(\mathcal{I}_{E}(d)\right)=0$ that is a contradiction. Thus $h^{1}\left(\mathcal{I}_{\operatorname{Res}_{L}(E)}(d-1)\right)>0$. Since $2 d+2-x \leq 2(d-1)+1$ ([6. Lemma 34]) gives the existence of a line $R$ such that $z:=\operatorname{deg}\left(R \cap \operatorname{Res}_{L}(E)\right) \geq d+1$. The maximality property of $x$ and the inclusion $\operatorname{Res}_{L}(E) \subseteq E$ gives $x \geq d+1$. Since $z \leq 2 d+2-x$, we get $z=x=d+1$. If $E$ has at least one reduced component, then $L \neq R$, because $\operatorname{deg}\left(\operatorname{Res}_{L}(E)\right)=$ $\operatorname{deg}(E \cap L)$ and $E$ has at least one reduced connected component. A Weil divisor $W$ of $L_{1} \cup L_{2}$ is locally principal, except at most at $L_{1} \cap L_{2}$. A well-known property of nodal singularities says that $W$ is Cartier if and only if $\operatorname{deg}(W)=\operatorname{deg}\left(W \cap L_{1}\right)+\operatorname{deg}\left(W \cap L_{2}\right)$. This condition is satisfied by $E$.

We remind here the part of [4, Theorem 1], that will be useful in our paper applied in the particular case of $\operatorname{deg}(Z)=4$.

Lemma 4. Assume $m \geq 2$ and let $E, F \subset \mathbb{P}^{m}$ be two 0-dimensional schemes such that there exists a point $Q \in\left\langle\nu_{m, d}(E)\right\rangle \cap\left\langle\nu_{m, d}(F)\right\rangle$ such that $Q \notin\left\langle\nu_{m, d}\left(E^{\prime}\right)\right\rangle$ for any $E^{\prime} \subsetneq E$ and $Q \notin\left\langle\nu_{m, d}\left(F^{\prime}\right)\right\rangle$ for any $F^{\prime} \subsetneq F$. Then there are a line $L \subset \mathbb{P}^{m}$ and a finite set of points $F_{2} \subset \mathbb{P}^{m} \backslash L$ such that $\operatorname{deg}(L \cap(E \cup F)) \geq d+2, E=F_{2} \sqcup(E \cap L)$ and $F=F_{2} \sqcup(F \cap L)$ (as schemes).

\section{Preliminaries of the proof of the main theorem}

This section is essentially the core of the proof of Theorem 1 but it is not the proof yet. Nevertheless that proof will be done in the next section. Here we give only all the preliminaries in such a way that the proof of Theorem 1 will be reduced to its structure.

Lemma 5. Let $E, F \subset \mathbb{P}^{m}$ be 0-dimensional schemes such that $h^{1}\left(\mathcal{I}_{E}(d)\right)=h^{1}\left(\mathcal{I}_{F}(d)\right)=0$. Let $M \subset \mathbb{P}^{m}$ be a hyperplane such that $h^{1}\left(\mathbb{P}^{m}, \mathcal{I}_{\operatorname{Res}_{M}(E \cup F)}(d-1)\right)=0$. Then $h^{1}\left(\mathbb{P}^{m}, \mathcal{I}_{E \cup F}(d)\right)=$ $h^{1}\left(M, \mathcal{I}_{(E \cup F) \cap M}(d)\right)$.

(a) If $\operatorname{Res}_{M}(E) \cap \operatorname{Res}_{M}(F)=\emptyset$, then $\left\langle\nu_{m, d}(E)\right\rangle \cap\left\langle\nu_{m, d}(F)\right\rangle=\left\langle\nu_{m, d}(E \cap M)\right\rangle \cap\left\langle\nu_{m, d}(E \cap M)\right\rangle \subseteq$ $\left\langle\nu_{m, d}(M)\right\rangle$.

(b) If $\operatorname{Res}_{M}(E) \cap \operatorname{Res}_{M}(F) \neq \emptyset$ and $F$ is reduced, then $\left\langle\nu_{m, d}(E)\right\rangle \cap\left\langle\nu_{m, d}(F)\right\rangle$ is the linear span of $\left\langle\nu_{m, d}(E \cap M)\right\rangle \cap\left\langle\nu_{m, d}(F \cap M)\right\rangle$ and of the set $\nu_{m, d}\left(\operatorname{Res}_{M}(E)_{\text {red }} \cap(F \backslash F \cup M)\right)$.

Proof. Since $h^{2}\left(Y_{m}, \mathcal{I}_{E \cup F}(d-1)\right)=0$, the first equality follows from the residual sequence (9) for the scheme $E \cup F$.

Assume $\operatorname{Res}_{M}(E) \cap \operatorname{Res}_{M}(F)=\emptyset$, i.e. $E \cap F \subset M$. Since $h^{1}\left(\mathbb{P}^{m}, \mathcal{I}_{E \cup F}(d)\right)=h^{1}\left(M, \mathcal{I}_{(E \cup F) \cap M}(d)\right)$, we have $\operatorname{dim}\left(\left\langle\nu_{m, d}(E \cup F)\right\rangle\right)=\operatorname{deg}(E \cup F)-1-h^{1}\left(\mathbb{P}^{m}, \mathcal{I}_{E \cup F}(d)\right)$, i.e. $\operatorname{dim}\left(\left\langle\nu_{m, d}(E)\right\rangle \cap\left\langle\nu_{m, d}(F)\right\rangle\right)=$ $\operatorname{deg}(E \cap F)-1+h^{1}\left(\mathbb{P}^{m}, \mathcal{I}_{E \cup F}(d)\right)$. For the same reason we have $\operatorname{dim}\left(\left\langle\nu_{m, d}(E \cap M)\right\rangle \cap\left\langle\nu_{m, d}(F \cap\right.\right.$ $M)\rangle)=\operatorname{deg}(E \cap F \cap M)-1+h^{1}\left(\mathbb{P}^{m}, \mathcal{I}_{E \cup F}(d)\right)$. Hence $\operatorname{dim}\left(\left\langle\nu_{m, d}(E)\right\rangle \cap\left\langle\nu_{m, d}(F)\right\rangle\right)=\operatorname{dim}\left(\left\langle\nu_{m, d}(E \cap\right.\right.$ $\left.M)\rangle \cap\left\langle\nu_{m, d}(F \cap M)\right\rangle\right)$. Hence $\left\langle\nu_{m, d}(E)\right\rangle \cap\left\langle\nu_{m, d}(F)\right\rangle=\left\langle\nu_{m, d}(E \cap M)\right\rangle \cap\left\langle\nu_{m, d}(F \cap M)\right\rangle$.

If $\operatorname{Res}_{M}(E) \cap \operatorname{Res}_{M}(F) \neq \emptyset$, then we need to add its degree to compute $\operatorname{dim}\left(\left\langle\nu_{m, d}(E)\right\rangle \cap\right.$ $\left.\left\langle\nu_{m, d}(F)\right\rangle\right)$. If $F$ is reduced, then $\operatorname{deg}\left(\operatorname{Res}_{M}(E) \cap \operatorname{Res}_{M}(F)\right)=\sharp\left(\operatorname{Res}_{M}(E)_{\text {red }} \cap(F \backslash F \cup M)\right)$.

Now we split the section in two subsections where we study the $X_{m, d}$-rank of a point $P \in$ $\left\langle\nu_{m, d}(A)\right\rangle$ for particular configurations of the scheme $A \subset \mathbb{P}^{m}$ with $m=2,3$ respectively (if $A \subset \mathbb{P}^{1}$ we refer to the Sylvester algorithm in [12, 6], 14] and [7 for the computation of the $X_{1, d}$-rank of a point $\left.P \in\left\langle\nu_{1, d}\left(\mathbb{P}^{1}\right)\right\rangle\right)$.

4.1. Two dimensional case. Here we study the $X_{2, d}$-rank of a point $P \in \sigma_{4}\left(X_{2, d}\right) \backslash\left(\sigma_{4}^{0}\left(X_{2, d}\right) \cup\right.$ $\left.\sigma_{3}\left(X_{2, d}\right)\right)$ with $X_{2, d}$ the Veronese surface $\nu_{2, d}\left(\mathbb{P}^{2}\right) \subset \mathbb{P}^{n_{2, d}}$. Moreover we assume in this sub-section that the scheme $A \subset \mathbb{P}^{2}$ such that $Z=\nu_{2, d}(A)$ evinces the $X_{2, d}$-border rank of $P$ is not contained in a line, that is to say that $m=2$ is the minimum dimension of a linear space that contains $A$ 
where $A$ is defined as in Notation 1 Since $A$ is not contained in a line we have that $\langle A\rangle=\mathbb{P}^{2}$ and $h^{0}\left(\mathbb{P}^{2}, \mathcal{I}_{A}(2)\right)=2$.

4.1.1. Here we assume the existence of a line $L \subset \mathbb{P}^{2}$ such that the schematic intersection between $A$ and $L$ has degree at least 3.

Since we are assuming that there exists a line $L \subset \mathbb{P}^{2}$ such that $\operatorname{deg}(A \cap L) \geq 3$ and since $A \nsubseteq L$ we have $\operatorname{deg}(A \cap L)=3$ and $\operatorname{Res}_{L}(A)$ is a point, $O$, with its reduced structure.

Notice that every point $P^{\prime}$ of $\left\langle\nu_{2, d}(A \cap L)\right\rangle \backslash \sigma_{2}\left(\nu_{2, d}(L)\right)$ has rank $d-1$ (12] or [24, Theorem 4.1]), unless $A \cap L$ is reduced. In the latter case any such a point has rank 3.

In Proposition 3 we study the case of $O \notin L$, while the case of $O \in L$ is done in Proposition 4.

Proposition 3. Let $A \subset \mathbb{P}^{2}$ be a 0-dimensional non-reduced scheme of degree 4. Assume the existence of a line $L \subset \mathbb{P}^{2}$ such that $\operatorname{deg}(L \cap A) \geq 3$ and $\operatorname{Res}_{L}(A)=:\{O\} \notin L$. Then

$$
r_{X_{2, d}}(P)=d
$$

for every $P \in\left\langle\nu_{2, d}(A)\right\rangle \backslash\left(\sigma_{4}^{0}\left(X_{2, d}\right) \cup \sigma_{3}\left(X_{2, d}\right)\right)$.

Proof. Since $\operatorname{Res}_{L}(A) \neq \emptyset$, we have $A \nsubseteq L$, i.e. $\operatorname{deg}(A \cap L)=3$. Hence $A=(A \cap L) \sqcup\{O\}$ with $O \notin L$. Since $P \notin\left\langle\nu_{d}(A \cap L)\right\rangle$, the line $\left\langle\left\{\nu_{2, d}(O), P\right\}\right\rangle \subset\left\langle\nu_{2, d}(A)\right\rangle$ meets the plane $\left\langle\nu_{2, d}(A \cap L)\right\rangle$ in a unique point $P^{\prime}$. We have $P^{\prime} \in\left\langle\nu_{2, d}(A \cap L)\right\rangle$, a theorem of Sylvester gives $P^{\prime} \in \sigma_{3}\left(\nu_{2, d}(L)\right) \backslash$ $\left(\sigma_{3}^{0}\left(\nu_{2, d}(L)\right) \cup \sigma_{2}\left(\nu_{2, d}(L)\right)\right)$ and $r_{\nu_{2, d}(L)}\left(P^{\prime}\right)=d-1$ (12, [14, [7, [24, Theorem 4.1], [6, §3]). Hence $r_{X}\left(P^{\prime}\right) \leq d-1$. Since $P \in\left\langle\left\{\nu_{2, d}(O), P^{\prime}\right\}\right\rangle$, we have $r_{X}(P) \leq r_{X}\left(P^{\prime}\right)+1=d$.

Assume $r_{X}(P)<d$, i.e. $\sharp(B) \leq d-1$. Hence $\operatorname{deg}(A \cup B) \leq d+3 \leq 2 d+1$. There is a line $R \subset \mathbb{P}^{2}$ such that $\operatorname{deg}((A \cup B) \cap R) \geq d+2(\underline{6}$, Lemma 34]).

First assume $R=L$. Since $P \notin\left\langle\nu_{2, d}(L)\right\rangle$ and $P \in\left\langle\nu_{m, d}(B)\right\rangle$, we have $B \cap L \subsetneq B$. Hence $\sharp(B \cap L) \leq d-2$. Hence $\operatorname{deg}(A \cap L) \geq 4$, a contradiction.

Now assume $R \neq L$. Since the scheme $R \cap L$ is a reduced point, we have $\operatorname{deg}(L \cap A \cap R) \leq 1$. Since $\operatorname{deg}(A \cap L)=\operatorname{deg}(A)-1$, we get $\operatorname{deg}(A \cap R) \leq 2$. Hence $\operatorname{deg}(R \cap B) \geq d$, contradicting the assumption $\sharp(B)<d$.

In the next two propositions we will do the cases in which the point $O=\operatorname{Res}_{L}(A)$ is contained in $L$. Observe that the definition of the residual scheme shows that the connected component $A_{O}$ of $A$ containing $O$ is not reduced. We will distinguish the three propositions below by the cardinality of the support of $A$.

Proposition 4. Let $A \subset \mathbb{P}^{2}$ be a 0-dimensional and connected scheme of degree 4 such that there is a line $L \subset \mathbb{P}^{2}$ with $\operatorname{deg}(L \cap A) \geq 3$ and $\operatorname{Res}_{L}(A)=:\{O\} \in L$. If $A$ is curvilinear, then

$$
r_{X_{2, d}}(P)=2 d-2
$$

for every $P \in\left\langle\nu_{2, d}(A)\right\rangle \backslash\left(\sigma_{4}^{0}\left(X_{2, d}\right) \cup \sigma_{3}\left(X_{2, d}\right)\right)$. If $A$ is not curvilinear, then $\left\langle\nu_{2, d}(A)\right\rangle \subset \sigma_{3}\left(X_{2, d}\right)$.

Proof. Since $\operatorname{Res}_{L}(A) \neq \emptyset$, we have $A \nsubseteq L$. Hence $\operatorname{deg}(A \cap L)=3$. If $A$ is not curvilinear, then we are in case (ii) of Lemma 1 with as $x$ a local equation of $L$. Hence $\left\langle\nu_{2, d}(A)\right\rangle \subset \sigma_{3}\left(X_{2, d}\right)$ (Lemma 11 and Remark 31). Hence from now on, we assume that $A$ is curvilinear. We first prove that $r_{X_{2, d}}(P) \geq 2 d-2$. Assume that $r_{X_{2, d}}(P) \leq 2 d-3$. Hence $\operatorname{deg}(A \cup B) \leq 2 d+2$. Therefore we may apply Lemma 4 Since $A_{r e d}$ is a single point, Lemma 4 gives $F_{2}=\emptyset$, i.e. $A \cup B \subset L$, a contradiction.

Now we prove that $r_{X_{2, d}}(P) \leq 2 d-2$. Let $L^{\prime} \subset \mathbb{P}^{2}$ be any line such that $O \in L^{\prime}$ and $L^{\prime} \neq L$. Since $\operatorname{Res}_{L}(A) \in L^{\prime}$ we have $A \subset L \cup L^{\prime}$. We will find $E \subset L \cup L^{\prime}$ such that $\sharp(E)=2 d-2$, $P \in\left\langle\nu_{2, d}(E)\right\rangle, \sharp(E \cap L)=d-1, \sharp\left(E \cap L^{\prime}\right)=d-1$ and $O \notin E$. Notice that $\left\langle\nu_{2, d}(L)\right\rangle$ and $\left\langle\nu_{2, d}\left(L^{\prime}\right)\right\rangle$ are $d$-dimensional linear subspaces of $\mathbb{P}^{n}$ with $\nu_{2, m}(O)$ as its intersection. Fix any $Q \in\left\langle\nu_{2, d}(A \cap L)\right\rangle$ such that $Q \notin\left\langle\nu_{2, d}(v)\right\rangle$, where $v$ is the tangent vector of $L$ at $O$. We have 
$r_{\nu_{2, d}(L)}(Q)=d-1\left(\left[12\right.\right.$, 24, Theorem 4.1], or [6, §3]). Fix $E_{1} \subset L$ such that $\nu_{2, d}\left(E_{1}\right)$ evinces $r_{\nu_{2, d}(L)}(Q)$. Since $P \notin\left\langle\nu_{2, d}(L)\right\rangle$ and $P \notin\left\langle\nu_{2, d}\left(L^{\prime}\right)\right\rangle$ ) (e.g. because the scheme $A$ is not contained in a line), the line $\langle\{Q, P\}\rangle$ meets $\left\langle\nu_{2, d}\left(L^{\prime}\right)\right\rangle$ at a unique point, $Q^{\prime}$. Take $E_{2} \subset L^{\prime}$ such that $\nu_{2, d}\left(E_{2}\right)$ evinces $r_{\nu_{2, d}\left(L^{\prime}\right)}\left(Q^{\prime}\right)$ and set $E:=E_{1} \cup E_{2}$. It is sufficient to prove that $\sharp\left(E_{2}\right) \leq d-1$. Assume $\sharp\left(E_{2}\right) \geq d$. Hence $\nu_{2, d}\left(Q^{\prime}\right)$ is contained in the tangent developable of $\nu_{2, d}\left(L^{\prime}\right)$ ([12], [24, Theorem 4.1], or [6, §3]). Hence there is a degree 2 scheme $W \subset L^{\prime}$ such that $Q^{\prime} \in\left\langle\nu_{2, d}(W)\right\rangle$. Hence $P \in\left\langle\nu_{2, d}\left(W \cup E_{1}\right)\right\rangle$. Since $\operatorname{deg}\left(\operatorname{Res}_{L}\left(W \cup E_{1} \cup A\right)\right) \leq 2$, we have $h^{1}\left(\mathcal{I}_{\operatorname{Res}_{L}\left(A \cup W \cup E_{1}\right)}(d-1)\right)=0$. Hence the proof of Lemma 5 gives $\operatorname{dim}\left(\left\langle\nu_{2, d}(A)\right\rangle \cap\left\langle\nu_{2, d}\left(W \cup E_{1}\right)\right\rangle\right)=\operatorname{dim}\left(\left\langle\nu_{2, d}(A \cap L) \cap\left\langle\nu_{2, d}((W \cup\right.\right.\right.$ $\left.\left.\left.\left.E_{1}\right) \cap L\right)\right\rangle\right)+\operatorname{deg}\left(A \cap\left(W \cup E_{1}\right)\right)-\operatorname{deg}\left((A \cap L) \cap\left(\left(W \cup E_{1}\right) \cap L\right)\right)$. We have $E_{1} \cap A=\emptyset$ and $\operatorname{deg}(W \cap L) \leq 1$, with equality only if $W_{\text {red }}=O$. Since $W \cap A=\emptyset$ if $W_{\text {red }} \neq O$, it is sufficient to notice that $\operatorname{deg}(W \cap A)=1$ if $W_{\text {red }}=O$, because $L^{\prime} \neq L$ and $L$ is the Zariski tangent space of the curvilinear scheme $A$.

Lemma 6. Let $D, R \subset \mathbb{P}^{2}$ be two different lines intersecting in $O \in \mathbb{P}^{2}$. Fix $P_{1} \in R \backslash\{O\}$ and let $A_{2} \subset R$ be the degree 2 effective divisor of $R$ with $P_{1}$ as its support. Let $A_{1} \subset D$ be the degree 2 effective divisor of $D$ with $O$ as its support. Set $A:=A_{1} \cup A_{2}$. Fix $P \in\left\langle\nu_{2, d}(A)\right\rangle$ such that $P \notin\left\langle\nu_{2, d}\left(A^{\prime}\right)\right\rangle$ for any $A^{\prime} \subsetneq A$. Then:

(a) $r_{X_{2, d}}(P)=2 d-2$.

(b) Fix any $B \subset \mathbb{P}^{2}$ evincing $r_{X_{2, d}}(P)$. Then $O \notin B, P_{1} \notin B, \sharp(B \cap R)=d-2$ and there is a line $L \subset \mathbb{P}^{2}$ such that $O \in L, L \neq D, R, \sharp(B \cap L)=d$ and $B \subset L \cup R$.

(c) Fix any line $L \subset \mathbb{P}^{2}$ such that $O \in L, L \neq D, R$. Then there is $B \subset \mathbb{P}^{2}$ evincing $r_{X_{2, d}}(P)$ and such that $\sharp(B \cap L)=d$.

Proof. Fix any line $L \subset \mathbb{P}^{2}$ passing through $O$ and such that $L \neq D, R$. We first prove the existence of a finite set $E \subset \mathbb{P}^{2}$ such that $\sharp(E)=2 d-2, O \notin E, P_{1} \notin E, \sharp(E \cap R)=d-2, \sharp(E \cap L)=d$ and $P \in\left\langle\nu_{2, d}(E)\right\rangle$. Let $2 O$ be the first infinitesimal neighborhood of $O$ in $\mathbb{P}^{2}$. Let $E_{1}, E_{2}, A_{1} \in \mathbb{P}^{2}$ be 0 -dimensional schemes obtained by intersecting $2 O$ with $L, R$ and $D$ respectively. Since $d \geq 4$, we have $\operatorname{dim}\left(\left\langle\nu_{2, d}\left(2 O \cup A_{2}\right)\right\rangle\right)=4$. Fix any $Q_{1} \in\left\langle\nu_{2, d}\left(E_{1}\right)\right\rangle \backslash\left\{\nu_{2, d}(O)\right\}$. Since $\left\langle\nu_{2, d}\left(E_{1}\right)\right\rangle$ is the tangent line at $\nu_{2, d}(O)$ of the rational normal curve $\nu_{2, d}(L)$, a theorem of Sylvester gives the existence of $B_{1} \subset L \backslash\{O\}$ such that $Q_{1} \in\left\langle\nu_{2, d}\left(B_{1}\right)\right\rangle$ ([12, [6], 7]). The 4-dimensional linear space $\left\langle\nu_{2, d}\left(2 O \cup A_{2}\right)\right\rangle$ contains $\left\langle\nu_{2, d}\left(E_{2} \cup A_{2}\right)\right\rangle$. Hence the line $\left\langle\left\{P, Q_{1}\right\}\right\rangle \subset\left\langle\nu_{2, d}\left(2 O \cup A_{2}\right)\right\rangle$ contains at least one point, $Q_{2}$, of $\left\langle\nu_{2, d}\left(E_{2} \cup A_{2}\right)\right.$.

Claim: For general $Q_{1} \in\left\langle\nu_{2, d}\left(E_{1}\right)\right\rangle \backslash\left\{\nu_{2, d}(O)\right\}$ we may find $Q_{2} \in\left\langle\left\{P, Q_{1}\right\}\right\rangle \cap\left\langle\nu_{2, d}\left(E_{2} \cup A_{2}\right)\right\rangle$ such that $Q_{2} \notin\left\langle\nu_{2, d}(F)\right\rangle$ for any $F \subsetneq E_{2} \cup A_{2}$.

Proof of the Claim: There are only 2 degree 3 subschemes of $E_{2} \cup A_{2}\left(\{O\} \cup A_{2}\right.$ and $\left.E_{2} \cup\left\{P_{1}\right\}\right)$ and it is sufficient to prove the claim for these subschemes. Assume the claim fails for one of them. Hence $\left\{P, Q_{1}\right\} \subset\left\langle\nu_{2, d}(F)\right\rangle$ with either $F=\{O\} \cup A_{2}$ or $F=E_{2} \cup\left\{P_{1}\right\}$. In particular $P \in\left\langle\nu_{2, d}(F)\right\rangle$. The case $F=\{O\} \cup A_{2}$ contradicts the hypothesis " $P \notin\left\langle\nu_{2, d}\left(A^{\prime}\right)\right\rangle$ for any $A^{\prime} \subsetneq A$ ", in fact $F$ is a proper subscheme of $A$. Now assume $F=E_{2} \cup\left\{P_{1}\right\}$. There is $Q_{4} \in\left\langle\nu_{2, d}\left(E_{2}\right)\right\rangle$ such that $P \in\left\langle Q_{4} \cup \nu_{2, d}\left(P_{1}\right)\right\rangle$. We get $r_{X_{2, d}}(P) \leq d+1$. The proof of parts (a) and (b) below (the line $D$ ) shows that $r_{X_{2} d}(P) \leq d+1$ is not even possible (alternatively the contradiction can be inferred from Lemma 4 because $d+1+4 \leq 2 d+1$ ).

By the Claim and the quoted theorem of Sylvester there is $B_{2} \subset R$ such that $\sharp\left(B_{2}\right)=d-2$ and $Q_{2} \in\left\langle\nu_{2, d}\left(B_{2}\right)\right\rangle$.

Set $E:=B_{1} \cup B_{2}$. Since $Q_{i} \in\left\langle\nu_{2, d}\left(B_{i}\right)\right\rangle, i=1,2, Q_{2} \in\left\langle\left\{P, Q_{1}\right\}\right\rangle$ and $Q_{1} \neq Q_{2}$, we have $P \in\left\langle\nu_{2, d}(E)\right\rangle$.

In particular we proved that $r_{X_{2, d}}(P) \leq 2 d-2$. Let $B \subset \mathbb{P}^{2}$ be a set evincing $r_{X_{2, d}}(P)$. Since $r_{X_{2, d}}(P) \leq 2 d-2$, we have $\operatorname{deg}(A \cup B) \leq 2 d+2$. 
First assume $\operatorname{deg}(A \cup B) \leq 2 d+1$. Since no component of $A$ is reduced, Lemma 4 gives that $A$ is contained in a line, a contradiction.

Now assume $\operatorname{deg}(A \cup B)=2 d+2$. Hence $A \cap B=\emptyset$. Since $h^{1}\left(\mathbb{P}^{2}, \mathcal{I}_{A \cup B}(d)\right)>0$ (Lemma 2), either there is a line $M \subset \mathbb{P}^{2}$ such that $\operatorname{deg}(M \cap(A \cup B)) \geq d+2$ or there is a conic $T$ such that $\operatorname{deg}(T \cap(A \cup B)) \geq 2 d+2$ (Lemma 3 ).

First assume the existence of a line $M$ such that $\operatorname{deg}(M \cap(A \cup B)) \geq d+2$. Since $\operatorname{deg}\left(\operatorname{Res}_{M}(A \cup\right.$ $B)) \leq d$, we have $h^{1}\left(\mathcal{I}_{\operatorname{Res}_{M}(A \cup B)}(d-1)\right)=0$. Since no connected component of $A$ is reduced, Lemma 4 gives $A \subset L$, a contradiction.

Now assume the existence of a conic $T$ such that $\operatorname{deg}(T \cap(A \cup B)) \geq 2 d+2$. Since $\operatorname{deg}(A \cup B) \leq$ $2 d+2$, we get $\operatorname{deg}(A \cup B)=2 d+2$ and $A \cup B \subset T$. Since $\operatorname{deg}(A \cup B)=2 d+2$, we have $O \notin B$ and $P_{2} \notin B$. Since $\operatorname{deg}(A \cap R)=3$, Bezout theorem gives $T=R \cup L$ with $L$ a line. Since $A_{1} \subset T$ and $A_{1} \not \subset R$, we have $O \in L$. We just checked that $\operatorname{deg}((A \cup B) \cap R) \leq d+1$ and $\operatorname{deg}((A \cup B) \cap L) \leq d-1$. Since $\operatorname{deg}\left(A_{1} \cap D\right)=2$, we get $L \neq D, \sharp(B \cap L)=d$ and $\sharp(B \cap R)=d-2$.

Corollary 1. Let $A \subset \mathbb{P}^{2}$ be a 0-dimensional scheme of degree 4 obtained as the union of two non-reduced degree 2 schemes $A_{1}, A_{2}$ with supports on the same line $R=\left\langle A_{2}\right\rangle$, and such that $D:=\left\langle A_{1}\right\rangle \neq R$. Set $O:=\left(A_{1}\right)_{\text {red }}$ and $O^{\prime}:=\left(A_{2}\right)_{\text {red }}$. Let $P \in\left\langle\nu_{2, d}(A)\right\rangle$ be such that $P \notin\left\langle\nu_{2, d}\left(A^{\prime}\right)\right\rangle$ for any $A^{\prime} \subsetneq A$. Set $Y:=\nu_{2, d}(R \cup L)$. Then

$$
r_{Y}(P)=2 d-1
$$

and there is $E \subset L \cup R$ such that $\nu_{2, d}(E)$ evinces $r_{Y}(P), O \notin E, O^{\prime} \notin E, \sharp(E \cap D)=d$ and $\sharp(E \cap R)=d-1$.

Proof. Lemma 6 gives $r_{X_{2, d}}(P)=2 d-2$ and that no set $B \subset \mathbb{P}^{2}$ evincing $r_{X_{2, d}}(P)$ is contained in $Y$. Hence it is sufficient to prove the existence of $E \subset L \cup R$ such that $\sharp(E)=2 d-1, O \notin E$, $O^{\prime} \notin E, \sharp(E \cap D)=d, \sharp(E \cap R)=d-1$ and $P \in\left\langle\nu_{2, d}(E)\right\rangle$. Fix a general $Q_{1} \in\left\langle\nu_{2, d}\left(A_{1}\right)\right\rangle$ and take $E_{1} \subset D \backslash\{O\}$ such that $Q_{1} \in\left\langle\nu_{2, d}\left(E_{1}\right)\right\rangle$ (Sylvester). Notice that $\left\langle\nu_{2, d}\left(\{O\} \cup A_{2}\right)\right\rangle$ is a hyperplane of the 3-dimensional linear space $\left\langle\nu_{2, d}(A)\right\rangle$. Hence the line $\left\langle\left\{Q_{1}, \nu_{2, d}\left(O^{\prime}\right)\right\}\right\rangle \subset\left\langle\nu_{2, d}(A)\right\rangle$ contains a point, $Q_{2}$, of $\left\langle\nu_{2, d}\left(\{O\} \cup A_{2}\right)\right\rangle$. Since $r_{X_{2, d}}(P)>d+2$, we have $Q_{2} \notin\left\langle\nu_{d}\left(\left\{O, O^{\prime}\right\}\right)\right\rangle$. It is easy to check that we may find $Q_{1}$ such that $Q_{2} \notin\left\langle\nu_{2, d}\left(A_{2}\right)\right\rangle$. Hence $r_{\nu_{2, d}(R)}\left(Q_{2}\right)=d-1$ (Sylvester). Take $B_{2} \subset R$ such that $\nu_{2, d}\left(B_{2}\right)$ evinces $r_{\nu_{2, d}(R)}\left(Q_{2}\right)$. Sylvester's theorem also gives $B_{2} \cap\left\{O, O^{\prime}\right\}=\emptyset$. Set $E:=B_{1} \cup B_{2}$.

Lemma 7. Fix a line $R \subset \mathbb{P}^{2}, O \in R$, and a connected 0-dimensional and curvilinear scheme $A \subset \mathbb{P}^{2}$ such that $\operatorname{deg}(A)=4, A_{\text {red }}=\{O\}$ and $\operatorname{deg}(R \cap A)=3$. Fix $P \in\left\langle\nu_{2, d}(A)\right\rangle$ such that $P \notin\left\langle\nu_{2, d}\left(A^{\prime}\right)\right\rangle$ for any $A^{\prime} \subsetneq A$. Then:

(a) $r_{X_{2, d}}(P)=2 d-2$.

(b) Fix any $B \subset \mathbb{P}^{2}$ evincing $r_{X_{2, d}}(P)$. Then $O \notin B$, $\sharp(B \cap R)=d-2$ and there is a line $L \subset \mathbb{P}^{2}$ such that $O \in L, L \neq R, \sharp(B \cap L)=d$ and $B \subset L \cup R$.

(c) Fix any line $L \subset \mathbb{P}^{2}$ such that $O \in L$ and $L \neq R$. Then there is $B \subset \mathbb{P}^{2}$ evincing $r_{X_{2, d}}(P)$ and such that $\sharp(B \cap L)=d$.

Proof. Let $A_{4} \subset R$ be the degree 4 effective divisor of $R$ with $O$ as its support. We modify the proof of Lemma 6 in the following way. Notice that the scheme $2 O \cup A_{4}$ has degree 5 . We use the 4-dimensional linear space $\left\langle\nu_{2, d}\left(2 O \cup A_{4}\right)\right\rangle$ instead of $\left\langle\nu_{2, d}\left(2 O \cup A_{2}\right)\right\rangle$ and the hyperplane $\left\langle\nu_{2, d}\left(A_{4}\right)\right\rangle$ of $\left\langle\nu_{2, d}(2 O \cup A)\right\rangle$ instead of $\left\langle\nu_{2, d}\left(E_{2} \cup A_{2}\right)\right\rangle$.

Proposition 5. Assume $d \geq 4$. Take $A=A_{O} \sqcup O_{1} \sqcup O_{2} \subset \mathbb{P}^{2}$ with $O_{1} \neq O_{2}$ two simple points of $\mathbb{P}^{2}$ and $A_{O} \subset \mathbb{P}^{2}$ a degree 2 non-reduced 0-dimensional scheme with support on a point $O \in L:=\left\langle O_{1}, O_{2}\right\rangle$ but $O \notin\left\{O_{1}, O_{2}\right\}$ and $\operatorname{deg}\left(A_{O} \cap L\right)=1$. Set $Z=\nu_{2, d}(A)$. Then

$$
r_{X_{2, d}}(P)=d+2
$$


for every $P \in\langle Z\rangle \backslash\left(\sigma_{4}^{0}\left(X_{2, d}\right) \cup \sigma_{3}\left(X_{2, d}\right)\right)$.

Proof. Define $Z_{O}:=\nu_{2, d}\left(A_{O}\right) \subset X_{2, d}$. Every point $P^{\prime} \in\left\langle\nu_{2, d}\left(Z_{O}\right)\right\rangle \backslash X_{2, d}$ has $X_{2, d}$-rank equal to $d$ (see [6, Theorem 32]). Thus $r_{X}(P) \leq d+2$ in this case. Assume $r_{X}(P) \leq d+1$. Since $d+5 \leq 2 d+1$ (here we are using the hypothesis $d \geq 4$ ), we may apply Lemma 4 . We get the existence of a line $R \subset Y_{2}$ and of a set of points $F_{2} \subset Y_{2} \backslash R$ such that $\operatorname{deg}((A \cap B) \cap R) \geq d+2$, $\sharp\left(F_{2}\right) \geq 1, B=\left(\left(B \backslash B \cap A_{\text {red }}\right) \cap R\right) \sqcup F_{2}, A \cap R \neq \emptyset, B \cap R \neq \emptyset, B=(B \cap R) \sqcup F_{2}$ and $A=(A \cap R) \sqcup F_{2}$ where $B$ is as in Notation 2. First assume $R=L$. Since $A_{\text {red }} \subset L$, we get $F_{2}=\emptyset$ and hence $A \subset L$, a contradiction. Now assume $R \neq L$. Thus $\{O\}=R \cap L, A_{O}$ is the degree 2 effective divisor of $R$ supported by $O$ and $F_{2}=\left\{O_{1}, O_{2}\right\}$. Since $P \notin\left\langle\nu_{2, d}\left(O_{1}\right), \nu_{2, d}\left(O_{2}\right), \nu_{2, d}(O)\right\rangle$ (in fact we have assumed that $Z=\nu_{2, d}(A)$ evinces the $X$-border rank of $P$ and $\operatorname{deg}(Z)=4$ ), we have $\left\langle\nu_{2, d}(A \cap L)\right\rangle \cap\left\langle\nu_{2, d}\left(A_{O}\right)\right\rangle \subseteq\langle(B \cap R) \backslash\{O\}\rangle$. Since $r_{\nu_{2, d}(R)}(U)=d$ for all $U \in\left\langle\nu_{2, d}\left(A_{O}\right)\right\rangle \backslash\{O\}$ (see [12]), we get $\sharp((B \cap R) \backslash\{O\}) \geq d$. Thus $\sharp(B) \geq d+2$, a contradiction.

Remark 4. Take $m \geq 2$ and $A=A_{O} \sqcup O_{1} \sqcup O_{2} \subset \mathbb{P}^{m}$ with $A_{O} \subset \mathbb{P}^{m}$ connected and $\operatorname{deg}\left(A_{O}\right)=2$ and $O_{1}, O_{2} \in \mathbb{P}^{m}$. Notice that if $m>2$ we are not assuming that $A$ is contained in a plane. As in Proposition 5 if $P \in\left\langle\nu_{m, d}\left(A_{O} \sqcup O_{1} \sqcup O_{2}\right)\right\rangle \backslash\left(\sigma_{4}^{0}\left(X_{m, d}\right) \cup \sigma_{3}\left(X_{m, d}\right)\right)$, then

$$
r_{X_{m, d}}(P)=d+2 .
$$

Let $L \subset \mathbb{P}^{m}$ be the line spanned by $A_{O}$. Set $\{O\}:=\left(A_{O}\right)_{\text {red }}$. Let $T$ be the tangent line to the degree $d$ rational normal curve $\nu_{m, d}(L)$ at $\nu_{m, d}(O)$. The plane $\left\langle\left\{\nu_{m, d}\left(O_{1}\right), \nu_{m, d}\left(O_{2}\right), P\right\}\right\rangle$ intersects $T$ at a unique point $P_{1}$ and $P_{1} \neq \nu_{m, d}(O)$. Hence $r_{\nu_{m, d}(L)}\left(P_{1}\right)=d$. Using Sylvester's algorithm (see [6, §3]) to find a set $S_{1} \subset \nu_{m, d}(L)$ evincing $r_{\nu_{m, d}(L)}\left(P_{1}\right)$. The set $S_{1} \cup\left\{\nu_{m, d}\left(O_{1}\right), \nu_{m, d}\left(O_{2}\right)\right\}$ evinces $r_{X_{m, d}}(P)$.

This concludes our Subsection 4.1.1 in which we were assuming the existence of a line $L \subset \mathbb{P}^{2}$ such that $\operatorname{deg}(A \cap L) \geq 3$.

4.1.2. Here we assume $\operatorname{deg}(A \cap L) \leq 2$ for every line $L \subset \mathbb{P}^{2}$. The assumption " $\operatorname{deg}(A \cap L) \leq 2$ for every line $L \subset \mathbb{P}^{2}$ " is equivalent to the spannedness of the sheaf $\mathcal{I}_{A}(2)$.

Let's do first the case in which the generic conic $E \in\left|\mathcal{I}_{A}(2)\right|$ is smooth.

Proposition 6. Assume $d \geq 4$. Let $A \subset \mathbb{P}^{2}$ be a zero dimensional scheme of degree 4 such that $\mathcal{I}_{A}(2)$ is spanned and $\sharp\left(A_{\text {red }}\right) \leq 2$. Suppose that the general conic $E \in\left|\mathcal{I}_{A}(2)\right|$ is smooth. Then

$$
r_{X_{2, d}(P)}=2 d-2
$$

for every $P \in\left\langle\nu_{2, d}(A)\right\rangle \backslash\left(\sigma_{4}^{0}\left(X_{2, d}\right) \cup \sigma_{3}\left(X_{2, d}\right)\right)$.

Proof. Notice that $Y:=\nu_{2, d}(E)$ is a degree $2 d$ rational normal curve in its linear span. Let $B \subset \mathbb{P}^{2}$ be defined as in Notation 2. Since $A \cup B \subset E$, we have $P \in\langle Y\rangle$. Since $r_{Y}(P)=2 d-2$ (see [12] or [24, Theorem 4.1]), we get $r_{X_{2, d}}(P) \leq 2 d-2$.

Assume $r_{X_{2, d}}(P) \leq 2 d-3$. Thus $\operatorname{deg}(A \cup B) \leq 2 d+1$. Take $L$ and $F_{2}$ as in the statement of Lemma 4. Since $\operatorname{deg}(L \cap E) \leq 2$, we have $\sharp\left(F_{2}\right) \geq 2$. Since $A$ is not reduced, we get $\sharp\left(A_{\text {red }}\right) \geq 3$, a contradiction.

Remark 5. Assume $m \geq 2$ and that the scheme $A \subset \mathbb{P}^{m}$ is as in Proposition 6, i.e. it is contained in a smooth conic $E \subset \mathbb{P}^{m}$ and $\sharp(A) \leq 2$. Set $Y:=\nu_{m, d}(E)$. In Proposition 6 we proved that $r_{Y}(P)=2 d-2$. Since one can use Sylvester's algorithm (see [6, §3]) to compute a set of points $S \subset C$ that evinces $r_{Y}(P)$, then one can use the same $S$ in order to compute $r_{X_{m, d}}(P)$, too.

Proposition 7. Let $A \subset \mathbb{P}^{2}$ be a non-reduced 0-dimensional scheme of degree 4 such that $\mathcal{I}_{A}(2)$ is spanned. Moreover suppose that a general conic $E \in\left|\mathcal{I}_{A}(2)\right|$ is not smooth: $E=L_{1} \cup L_{2}$ with 
$L_{1}$ and $L_{2}$ lines and $L_{1} \neq L_{2}$. Assume $\sharp\left(A_{\text {red }}\right)=3$. Fix any $P \in\left\langle\nu_{2, d}(A)\right\rangle \backslash\left(\sigma_{4}^{0}\left(X_{2, d}\right) \cup \sigma_{3}\left(X_{2, d}\right)\right)$. If $d=3$, then $r_{X_{2, d}}(P)=4$. If $d \geq 4$, then

$$
r_{X_{2, d}}(P)=d+2 .
$$

Proof. Let $A_{O}$ be the non-reduced connected component of $A$ and $O_{1}, O_{2}$ the reduced ones. Since $\nu_{2, d}\left(A_{O}\right)$ is a tangent vector of $X_{2, d}, r_{X_{2, d}}\left(P^{\prime}\right)=d$ for all $P^{\prime} \in\left\langle\nu_{2, d}\left(A_{O}\right)\right\rangle \backslash\left(A_{O}\right)_{\text {red }}$ ( $\underline{6}$. Theorem $32])$. Thus $r_{X_{2, d}}(P) \leq d+2$. First assume $d \geq 4$. Using Lemma 4 we easily get that $r_{X_{2, d}}(P) \geq d+2$ (see Proposition [5 for a similar case). If $d=3$, then we use [6. Theorem 40] or [22, Remark 2.3.1] and get the inequality $r_{X_{2,3}}(P) \geq 4$.

Remark 6. Observe that in this Section 2 we are assuming that $\mathcal{I}_{A}(2)$ is spanned, this implies that a general $E \in\left|\mathcal{I}_{A}(2)\right|$ is reduced (Bertini's theorem) and $A$ is the complete intersection of two general elements of $\left|\mathcal{I}_{A}(2)\right|$.

Lemma 8. Assume $\mathcal{I}_{A}(2)$ spanned (equivalently, assume $\operatorname{deg}(A \cap L) \leq 2$ for every line $L \subset \mathbb{P}^{2}$ ) and that a general $E \in\left|\mathcal{I}_{A}(2)\right|$ is not smooth. Then $A$ is connected, a complete intersection and not curvilinear.

Proof. Since $E$ is not a double line (Remark 6), $E$ has a unique singular point. Call it $Q$. Since $\mathcal{I}_{A}(2)$ is spanned, $Q \in A_{\text {red }}$ (Bertini's theorem). Since $\left|\mathcal{I}_{A}(2)\right|$ is a pencil (and hence it is irreducible as an abstract variety) and $A_{r e d}$ is finite, $Q \in \operatorname{Sing}(F)$ for every $F \in\left|\mathcal{I}_{A}(2)\right|$. Take a general $F \in\left|\mathcal{I}_{A}(2)\right| \backslash\{E\}$. Both $E$ and $F$ are reduced (Remark 6 ). Since no line is in the base locus of $\left|\mathcal{I}_{A}(2)\right|, E$ and $F$ have no common component. Since $\operatorname{deg}(A)=4$, we get $A=E \cap F$ (schemetheoretic intersection). Hence $A_{\text {red }}=\{P\}$ and $A$ is isomorphic to the scheme defined around $(0,0) \in \mathbb{A}^{2}$ by two quadratic forms in two variables. Hence the Zariski tangent space of $A$ at $Q$ has dimension two.

Proposition 8. Let $A \subset \mathbb{P}^{2}$ be a non-reduced zero dimensional scheme of degree 4 such that $\mathcal{I}_{A}(2)$ is spanned and a general $E \in\left|\mathcal{I}_{A}(2)\right|$ is not smooth. Write $E=L_{1} \cup L_{2}$ with $L_{1}$ and $L_{2}$ lines and $L_{1} \neq L_{2}$.

Assume $\sharp\left(A_{\text {red }}\right)=1$. Then

$$
r_{X_{2, d}}(P)=2 d-2
$$

for every $P \in\left\langle\nu_{2, d}(A)\right\rangle \backslash\left(\sigma_{4}^{0}\left(X_{2, d}\right) \cup \sigma_{3}\left(X_{2, d}\right)\right)$.

Proof. Since $\operatorname{deg}\left(A \cap L_{i}\right) \leq 2$ for all $i$, we have $A_{\text {red }}=L_{1} \cap L_{2}$. Since $A$ is a Cartier divisor of $E$, we may apply Proposition 1. Thus $r_{\nu_{2, d}(E)}(P)=2 d-2$. Hence $r_{X_{2, d}}(P) \leq 2 d-2$. Thus it is sufficient to prove $r_{X_{2, d}}(P) \geq 2 d-2$. Assume $r_{X_{2, d}}(P) \leq 2 d-3$. Hence $\operatorname{deg}(A \cup B) \leq 2 d+1$ for $B$ as in Notation 2. Thus we may apply Lemma 4 . Since $A$ is connected, $F_{2}=\emptyset$, contradicting the inequality $\sharp\left(F_{2}\right) \geq m-1=1$.

Now we can prove that the inequality in Proposition 1 is actually an equality.

Corollary 2. Fix an integer $d \geq 3$. Let $Y \subset \mathbb{P}^{2 d}$ be a reduced and connected curve union of two smooth degree $d$ curves $Y_{1}, Y_{2}$, each of them a rational normal curve in its linear span, with a unique common point point, $Q$, and with $\langle Y\rangle=\mathbb{P}^{2 d}$. Let $Z \subset Y$ be a 0-dimensional scheme such that $\operatorname{deg}(Z)=4, Z_{\text {red }}=\{Q\}, Z$ is a Cartier divisor of $Y$ and $\operatorname{deg}\left(Z \cap Y_{i}\right) \geq 2$ for $i=1,2$. Fix $P \in\langle Z\rangle$ such that $P \notin\left\langle Z^{\prime}\right\rangle$ for any $Z^{\prime} \subsetneq Z$. Then

$$
r_{Y}(P)=2 d-2 .
$$

Proof. The inequality $r_{Y}(P) \leq 2 d-2$ is proved in Proposition 1 .

In the proof of Proposition 4 we showed that if $\operatorname{deg}\left(A \cap L_{i}\right)=3$ for one $i=1,2$ (i.e. if $\operatorname{deg}\left(Z \cap Y_{i}\right) \geq 2$ for one $i=1,2)$, then $r_{X_{2, d}}(P) \geq 2 d-2$. Since $Y \subseteq X_{2, d}$, we have $r_{Y}(P) \geq r_{X_{2, d}}(P) \geq 2 d-2$, concluding the proof. 
4.2. Three dimensional case. Here we assume that $m=3$ and that the degree 4 non-reduced 0 -dimensional scheme $A \subset \mathbb{P}^{3}$ introduced in Notation 1 is not contained in any plane of $\mathbb{P}^{3}$, i.e., $\operatorname{dim}(\langle A\rangle)=3$.

Remark 7. If $A \subset \mathbb{P}^{3}$ is the first infinitesimal neighborhood $2 Q$ of some point $Q \in \mathbb{P}^{3}$ then, if $Z \subset X_{3, d}$ is as in Notation 1 the linear span $\langle Z\rangle$ is actually the tangent space $T_{\nu_{3, d}(Q)} X_{3, d}$ of $X_{3, d}$ at $\nu_{3, d}(Q)$. Therefore, by [6, Theorem 32], we have $r_{X_{3, d}}(P)=d$, but also that $P \in \sigma_{2}\left(X_{3, d}\right)$.

Proposition 9. Assume $d \geq 3$. Let $U_{1}, U_{2} \subset \mathbb{P}^{3}$ be two disjoint non-reduced 0-dimensional schemes of degree 2 such that $A:=U_{1} \sqcup U_{2}$ spans $\mathbb{P}^{3}$. Then

$$
r_{X_{3, d}}(P)=2 d
$$

for every $P \in\left\langle\nu_{3, d}(A)\right\rangle \backslash\left(\sigma_{4}^{0}\left(X_{3, d}\right) \cup \sigma_{3}\left(X_{3, d}\right)\right)$.

Proof. Proposition 2 gives $r_{X_{3, d}}(P) \leq 2 d$. Here we will prove the reverse inequality and hence that $r_{X_{3, d}}(P)=2 d$ for $d \geq 3$. Assume $r_{X_{3, d}}(P) \leq 2 d-1$ and take $B \subset X_{3, d}$ such that $\nu_{3, d}(B)$ evinces $r_{X_{3, d}}(P)$. By assumption we have $\operatorname{deg}(A \cup B) \leq 2 d+3$. Let $M \subset \mathbb{P}^{3}$ be a plane such that $\operatorname{deg}(M \cap(A \cup B))$ is maximal. Consider the residual exact sequence (9) with $t=d, H=M$ and $E=A \cup B$. Since $h^{1}\left(\mathbb{P}^{3}, \mathcal{I}_{A \cup B}(d)\right)>0$ (Lemma 2), we get that either $h^{1}\left(\mathcal{I}_{\operatorname{Res}_{M}(A \cup B)}(d-1)\right)>0$ or $h^{1}\left(M, \mathcal{I}_{M \cap(A \cup B}(d)\right)>0$. Since $h^{1}\left(\mathbb{P}^{3}, \mathcal{I}_{A \cup B}(d)\right)>0$ and $\operatorname{deg}(A \cup B) \leq 2 d+3 \leq 3 d+1, A \cup B$ is not in linearly general position ([18, Theorem 3.2]). Hence $\operatorname{deg}(M \cap(A \cup B)) \geq 4$.

(a) In this step and in the next one we assume $h^{1}\left(M, \mathcal{I}_{(A \cup B) \cap M, M}(d)\right)>0$, i.e. $\left\langle\nu_{3, d}(A \cap\right.$ $M)\rangle \cap\left\langle\nu_{3, d}(B \cap M)\right\rangle \neq \emptyset$. In this step we also assume $\operatorname{deg}((A \cup B) \cap M) \geq 2 d+2$. Since $A \cup B$ spans $\mathbb{P}^{3}$ and $\operatorname{deg}(A \cup B) \leq 2 d+3$, we get $\operatorname{deg}((A \cup B) \cap M)=2 d+2$ and that $\operatorname{Res}_{M}(A \cup B)$ is a reduced point, say $Q$. Since $P \in\langle Z\rangle \cap\langle S\rangle$, to compute $r_{X_{3, d}}(P)$ we cannot use a smaller number of variables (see [23, Exercise 3.2.2.2], or [10, Theorem 2.1], for a generalization in the non-symmetric case). Thus $Q \in A_{\text {red }} \cap B_{\text {red }}$. Thus $\operatorname{deg}(A \cup B) \leq \operatorname{deg}(A)+\operatorname{deg}(B \backslash\{Q\}) \leq 2 d+2$. Since $\operatorname{deg}((A \cup B) \cap M) \geq 2 d+2$, we get $A \cup B \subset M$, a contradiction.

(b) Here we assume $h^{1}\left(M, \mathcal{I}_{(A \cup B) \cap M, M}(d)\right)>0$ and $\operatorname{deg}((A \cup B) \cap M) \leq 2 d+1$. Since $h^{1}\left(M, \mathcal{I}_{(A \cup B) \cap M, M}(d)\right)>0$ and $\operatorname{deg}((A \cup B) \cap M) \leq 2 d+1$, there is a line $L$ such that $\sharp((A \cup$ $B) \cap L) \geq d+2\left(\left[\right.\right.$, Lemma 34]). Since $\nu_{3, d}(B)$ is linearly independent, we have $\sharp(B \cap L) \leq d+1$. Since $A$ spans $\mathbb{P}^{3}$ and $\operatorname{deg}(A)=4$, we have $\operatorname{deg}(A \cap R) \leq 2$ for every line $R \subset \mathbb{P}^{3}$. Therefore $\operatorname{deg}(A \cap L) \leq 2$ and $d \leq \operatorname{deg}(B \cap L) \leq d+1$. Assume for the moment $\operatorname{Res}_{M}(A) \cap \operatorname{Res}_{M}(B)=\emptyset$. Since $P \notin\left\langle\nu_{3, d}(M)\right\rangle$, Lemma 5 gives $h^{1}\left(\mathbb{P}^{3}, \mathcal{I}_{\operatorname{Res}_{M}(A \cup B)}(d-1)\right)>0$. Hence $\operatorname{deg}\left(\operatorname{Res}_{M}(A \cup B)\right) \geq d+1$. Since $\operatorname{deg}(A \cup B) \leq 2 d+2$ and $\operatorname{deg}((A \cup B) \cap M) \geq d+2$, we obtained a contradiction. Now assume $\operatorname{Res}_{M}(A) \cap \operatorname{Res}_{M}(B) \neq \emptyset$. Since $\nu_{3, d}(B)$ is linearly independent, we must have $A \cap M \neq \emptyset$. Hence $M$ meets exactly one of the connected components of $A$ and $B$ contains the support of the other connected component of $A$, say $\left(U_{1}\right)_{\text {red }} \in M,\left(U_{2}\right)_{\text {red }} \notin M$ and $\left(U_{2}\right)_{\text {red }} \in B$. Lemma 5 gives that $P$ is the linear span of $\left\langle\nu_{3, d}(A \cap M)\right\rangle$ and the point $\nu_{3, d}\left(\left(U_{2}\right)_{r e d}\right)$. Hence $P \in \sigma_{3}\left(X_{3, d}\right)$, a contradiction.

(c) Now assume $h^{1}\left(M, \mathcal{I}_{(A \cup B) \cap M, M}(d)\right)=0$. Hence $h^{1}\left(\mathcal{I}_{\operatorname{Res}_{M}(A \cup B)}(d)\right)>0$ by the residual exact sequence. Since $\operatorname{deg}((A \cup B) \cap M) \geq 4$, we have $\operatorname{deg}\left(\operatorname{Res}_{M}(A \cup B)\right) \leq 2(d-1)+1$. Hence [6. Lemma 34], gives the existence of a line $L \subset \mathbb{P}^{3} \operatorname{such}$ that $\operatorname{deg}\left(L \cap \operatorname{Res}_{M}(A \cup B)\right) \geq(d-1)+2$. Since $\operatorname{Res}_{M}(A \cup B) \subseteq A \cup B$, we get $\operatorname{deg}((A \cup B) \cap L) \geq d+1$. Since $h^{1}\left(M, \mathcal{I}_{(A \cup B) \cap M, M}(d)\right)=0$, we have $\operatorname{deg}((A \cup B) \cap L)=d+1$. Let $N \subset \mathbb{P}^{3}$ be a general plane containing $L$. Since $A \cup B$ is curvilinear and $(A \cup B)_{\text {red }}$ is finite, we have $N \cap(A \cup B)=L \cap(A \cup B)$ (as schemes). Hence $h^{1}\left(N, \mathcal{I}_{N \cap(A \cup B)}(d)\right)=0$. The residual exact sequence of $N$ gives $h^{1}\left(\mathcal{I}_{\operatorname{Res}_{N}(A \cup B)}(d-1)\right)>0$. Since $\operatorname{deg}\left(\operatorname{Res}_{N}(A \cup B)\right) \leq 2 d+3-d-1 \leq 2(d-1)+1$, there is a line $T \subset \mathbb{P}^{3}$ such that $\operatorname{deg}\left(T \cap \operatorname{Res}_{N}(A \cup B)\right) \geq d+1$. Since $B$ is reduced and $L \subset N$, we have $T \nsubseteq N$ and in particular 
$T \neq L$. Hence $\sharp(B \cap T \backslash B \cap T \cap L) \geq d-2$. Fix any $o \in B \cap T \backslash(T \cap B \cap T \cap L)$. Let $N_{o}$ be the plane spanned by $L$ and $o$. If $\operatorname{deg}\left(N_{o} \cap(A \cup B)\right) \geq 2 d+2$, part (a) gives a contradiction. Hence we may assume $\operatorname{deg}\left(N_{o} \cap(A \cup B)\right) \leq 2 d+1$. First assume $h^{1}\left(N_{o}, \mathcal{I}_{N_{o} \cap(A \cup B)}(d)\right)=0$. The residual sequence of $N_{o}$ gives $h^{1}\left(\mathcal{I}_{\operatorname{Res}_{N_{o}}(A \cup B)}(d-1)\right)>0$. Hence there is a line $T_{o}$ such that $\operatorname{deg}\left(T_{o} \cap \operatorname{Res}_{N_{o}}(A \cup B)\right) \geq d+1$.We may assume $\operatorname{deg}\left(T_{o} \cap(A \cup B)\right)=d+1$ (e.g. by parts (a) and (b)). Since $B$ is reduced, and $o \in B \cap N_{o}$, we have $o \notin T_{o}$. Since $N_{o} \supset L$, we have $T_{o} \neq L$. We get $\operatorname{deg}(A \cup B) \geq 3(d+1)-2$, a contradiction. Now assume $h^{1}\left(N_{o}, \mathcal{I}_{N_{o} \cap(A \cup B)}(d)\right)>0$. Since $\operatorname{deg}\left(N_{o} \cap(A \cup B) \leq 2 d+1\right.$, there is a line $D_{o} \subset N_{o}$ such that $\operatorname{deg}\left(D_{o} \cap(A \cup B)\right) \geq d+2$. Hence $D_{o} \neq L$ and $D_{o} \neq T$. The lines $L, T$ and $D_{o}$ give $\operatorname{deg}(A \cup B)>2 d+3$, a contradiction.

Remark 8. Assume, for $m>2$, that the 0 -dimensional scheme $A \subset \mathbb{P}^{m}$ of Notation 1 has two connected components, $A_{1}, A_{2} \subset \mathbb{P}^{m}$, both of degree 2 and that the lines $L_{i}:=\left\langle A_{i}\right\rangle, i=1,2$, are disjoint. Thus $\operatorname{dim}\left(\left\langle L_{1} \cup L_{2}\right\rangle\right)=3$. Set $Y_{i}:=\nu_{m, d}\left(L_{i}\right), i=1,2$, and $Y:=Y_{1} \cup Y_{2}$. Notice that $Y_{1} \cap Y_{2}=\emptyset$. Now let $Z \subset X_{m, d}$ be defined as in Notation 1 as a scheme that evinces the $X_{m, d}$-border rank of a point $P \in\langle Z\rangle \backslash\left(\sigma_{4}^{0}\left(X_{m, d}\right) \cup \sigma_{3}\left(X_{m, d}\right)\right)$. By [25, Proposition 3.1], or 24, Subsection 3.2], $r\left(X_{m, d}\right)(P)=r_{Y_{1} \cup Y_{2}}(P)$. We proved in Proposition 2 that $r_{X_{m, d}}(P)=2 d$ and that it may be evinced by a set $S \subset Y$ such that $\sharp\left(S \cap Y_{i}\right)=d, i=1,2$. The set $S$ may be found in the following way (here we just translate the proof of Proposition 2):

Step 1. Set $P_{2}:=\left\langle\{P\} \cup Y_{1}\right\rangle \cap\left\langle Y_{2}\right\rangle$ and $P_{1}:=\left\langle\{P\} \cup Y_{2}\right\rangle \cap\left\langle Y_{1}\right\rangle$.

Step 2. Find $S_{i} \subset Y_{i}$ evincing the $Y_{i}$-rank of $P_{i}$ (e.g. use Sylvester's algorithm [12, [6], [14] and [7]).

Step 3. Set $S:=S_{1} \cup S_{2}$.

Proposition 10. Assume $d \geq 3$. Let $A \subset \mathbb{P}^{3}$ be a degree 4 curvilinear 0-dimensional scheme with support at only one point and such that $\langle A\rangle=\mathbb{P}^{3}$. Then

$$
r_{X_{3, d}}(P)=3 d-2
$$

for all $P \in\left\langle\nu_{3, d}(A)\right\rangle \backslash\left(\sigma_{4}^{0}\left(X_{m, d}\right) \cup \sigma_{3}\left(X_{3, d}\right)\right)$.

Proof. Since $A$ spans $\mathbb{P}^{3}$, it is projectively equivalent to a connected degree 4 divisor of a smooth rational normal curve $Y$ of $\mathbb{P}^{3}$. Thus $r_{X_{3, d}}(P) \leq r_{\nu_{3, d}(Y)}(P)=3 d-2$ (12]). In order to obtain a contradiction we assume $r_{X_{3, d}}(P) \leq 3 d-3$.

Take $B \subset \mathbb{P}^{3}$ such that $\nu_{3, d}(B)$ evinces $r_{X_{3, d}}(P)$. We have $\operatorname{deg}(A \cup B)=4+r_{X_{3, d}}(P)-\operatorname{deg}(A \cap$ $B) \leq 3 d+1$. Lemma 2 gives $h^{1}\left(\mathbb{P}^{m}, \mathcal{I}_{A \cup B}(d)\right)>0$. Hence $A \cup B$ is not in linearly general position (see [18, Theorem 3.2]). Thus there is a plane $M \subset \mathbb{P}^{3}$ such that $\operatorname{deg}(M \cap(A \cup B)) \geq 4$. Among all such planes we take one, say $M_{1}$, such that $x_{1}:=\operatorname{deg}\left(M_{1} \cap(A \cup B)\right)$ is maximal. Set $E_{1}:=A \cup B$ and $E_{2}:=\operatorname{Res}_{M_{1}}\left(E_{1}\right)$. Notice that $\operatorname{deg}\left(E_{2}\right)=\operatorname{deg}\left(E_{1}\right)-x_{1}$. Define inductively the planes $M_{i} \subset \mathbb{P}^{3}, i \geq 2$, the schemes $E_{i+1}, i \geq 2$, and the integers $x_{i}, i \geq 2$, by the condition that $M_{i}$ is one of the planes such that $x_{i}:=\operatorname{deg}\left(M_{i} \cap E_{i}\right)$ is maximal and then set $E_{i+1}:=\operatorname{Res}_{M_{i}}\left(E_{i}\right)$. We have $E_{i+1} \subseteq E_{i}$ (with strict inclusion if $E_{i} \neq \emptyset$ ) for all $i \geq 1$ and $E_{i}=\emptyset$ for all $i \gg 0$. For all integers $t$ and $i \geq 1$ there is the residual exact sequence

$$
0 \rightarrow \mathcal{I}_{E_{i+1}}(t-1) \rightarrow \mathcal{I}_{E_{i}}(t) \rightarrow \mathcal{I}_{E_{i} \cap M_{i}, M_{i}}(t) \rightarrow 0
$$

Let $z$ be the minimal integer $i$ such that $1 \leq i \leq d+1$ and $h^{1}\left(M_{i}, \mathcal{I}_{M_{i} \cap E_{i}}(d+1-i)\right)>0$. Use at most $d+1$ times the exact sequences (10) to prove the existence of such an integer $z$. We now study the different possibilities that we have for the integer $z$ just defined.

(a) Here we assume $z=1$. Since $\nu_{3, d}(B)$ is linearly independent and $h^{1}\left(M_{1}, \mathcal{I}_{(A \cup B) \cap M_{1}}(d)\right)>$ 0 , we have $A_{\text {red }} \in M_{1}$. Since $B$ is reduced, we get $\operatorname{Res}_{M_{1}}(A) \cap \operatorname{Res}_{M_{1}}(B)=\emptyset$. Since $P \notin$ $\left\langle\nu_{3, d}\left(M_{1}\right)\right\rangle$, Lemma 5 gives $h^{1}\left(\mathbb{P}^{3}, \mathcal{I}_{E_{2}}(d-1)\right)>0$. Hence $x_{2} \geq d+1$. Since by hypothesis $d \geq 3$, $x_{2} \leq x_{1}$ and $x_{1}+x_{2} \leq 3 d+1$, we have $x_{2} \leq 2 d-1$. Hence there is a line $R \subset \mathbb{P}^{3}$ such that 
$\operatorname{deg}\left(E_{2} \cap R\right) \geq d+1$ ([6, Lemma 34]). Hence $x_{1} \leq 2 d$. Since $h^{1}\left(M_{1}, \mathcal{I}_{(A \cup B) \cap M_{1}}(d)\right)>0$, 6. Lemma 34], gives the existence of a line $L \subset M_{1} \operatorname{such}$ that $\operatorname{deg}(L \cap(A \cup B)) \geq d+2$. Since $\nu_{3, d}(B)$ is linearly independent, we get $A \cap L \neq \emptyset$. Since $B$ is reduced, $\operatorname{deg}(A \cap T) \leq 2$ for any line $T \subset \mathbb{P}^{3}$ and $\operatorname{deg}\left(R \cap \operatorname{Res}_{M_{1}}(A \cup B)\right) \geq 3$, we have $R \nsubseteq M_{1}$ and in particular $R \neq L$. First assume $R \cap L \neq \emptyset$. Since $\operatorname{deg}((A \cup B) \cap\langle L \cup R\rangle) \geq 2 d+1$, we have $x_{1} \geq 2 d+1$. Hence $x_{2} \leq d$, a contradiction. Now assume $L \cap R=\emptyset$. In particular we have $A_{\text {red }} \notin R$. Hence $\sharp(R \cap B) \geq d+1$. Since $\nu_{3, d}(B)$ is linearly independent, we have $\sharp(R \cap B)=d+1$. Fix any $Q \in R \cap B$. Let $H \subset \mathbb{P}^{3}$ be the plane spanned by $L$ and by $Q$. Since $A_{\text {red }} \in L$ and $B$ is reduced, we have $\operatorname{Res}_{H}(A) \cap \operatorname{Res}_{H}(B)=\emptyset$. Lemma 5 gives $h^{1}\left(\mathcal{I}_{\operatorname{Res}_{H}(A \cup B)}(d-1)\right)>0$. Since $\operatorname{deg}((A \cup B) \cap H) \geq d+3$, we have $\operatorname{deg}\left(\operatorname{Res}_{H}(A \cup B)\right) \leq 2 d-2 \leq 2(d-1)+1$. Hence there is a line $R^{\prime} \subset \mathbb{P}^{3}$ such that $\operatorname{deg}\left(R^{\prime} \cap\left(\operatorname{Res}_{H}(A \cup B)\right) \geq d+1\right.$. Since $L \subset H$ and $B$ is reduced, we have $R^{\prime} \neq L$. Since $\operatorname{deg}((A \cup B) \cap R)=d+1$ and $H$ contains one of the points of $R$, we have $R^{\prime} \neq R$. If $R^{\prime} \cap L \neq \emptyset$, using the plane $\left\langle L \cup R^{\prime}\right\rangle$ we get $x_{1} \geq 2 d+1$ and $x_{2} \geq d+1$, a contradiction. If $R^{\prime} \cap L=\emptyset$, then $\operatorname{deg}(A \cup B) \geq d+2+2 d+1$, a contradiction.

(b) From now on we assume $z>1$. Since $h^{1}\left(M_{z}, \mathcal{I}_{M_{z} \cap E_{z}}(d+1-z)\right)>0$, we have $x_{z}:=$ $\operatorname{deg}\left(M_{z} \cap E_{z}\right) \geq d+3-z$. Since the function $z \mapsto x_{z}$ is non-increasing, we get $x_{i} \geq d+3-z$ for all $i \in\{1, \ldots, z+1\}$. Since $\operatorname{deg}(A \cup B) \geq z(d+3-z)$, we get $3 d+1 \geq z(d+3-z)$. Hence either $z \in\{2,3\}$ or $z \geq d$ (this statement is trivially true if $d=3,4$ ).

(c) Assume $z=d$. The condition $h^{1}\left(\mathcal{I}_{M_{d} \cap E_{d}}(1)\right)>0$ says that either $M_{d} \cap E_{d}$ contains a scheme of length $\geq 3$ contained in a line $R$ or $x_{d} \geq 4$. If $x_{d} \geq 4$, then we get $x_{1}+\cdots+x_{d} \geq 4 d$, that is a contradiction. Hence we may assume $x_{1}=4, x_{i}=3$ for $2 \leq i \leq d$ and $x_{d+1}=0$. Since $x_{2}=3$, the maximality of the integer $x_{2}$ gives that $E_{2}$ is in linearly general position. Since $\operatorname{deg}\left(E_{2}\right)=\operatorname{deg}\left(E_{1}\right)-x_{4} \leq 3(d-1)+1$ and $E_{2}$ is in linearly general position, then $h^{1}\left(\mathcal{I}_{E_{2}}(d-1)\right)=0$. Since $z>1$. $h^{1}\left(M_{1}, \mathcal{I}_{E_{1} \cap M_{1}}(d)\right)=0$. Hence (10) with $i=1$ and $t=d$ gives a contradiction.

(d) Assume $z=d+1$. The condition $h^{1}\left(M_{i}, \mathcal{I}_{M_{z} \cap E_{z}}\right)>0$ only says $x_{d+1} \geq 2$. Taking the first integer $y \leq d$ such that $x_{y} \leq 3$ and $E_{y}$ is not collinear, we get a contradiction as above.

(e) Assume $z=2$. Since $3 d+1 \geq x_{1}+x_{2} \geq 2 x_{2}$, we get $x_{2} \leq 2(d-1)+1$. By [6. Lemma 34], there is a line $R \subset M_{2}$ such that $\left.\operatorname{deg}\left(\operatorname{Res}_{M_{1}}(A \cup B) \cap R\right)\right) \geq d+1$. Hence $x_{2} \geq d+1$. Since $x_{2} \geq d+1$, we have $x_{1} \leq 2 d$. Since $\operatorname{Res}_{M_{1}}(B) \cap R \neq \emptyset$ and $B$ is reduced, we have $R \nsubseteq M_{1}$. Since $z>1$ and $R \subset M_{1}$, we get $\operatorname{deg}((A \cup B) \cap R)=d+1$. Let $H$ be a plane containing $R$ and such that $e_{1}:=\operatorname{deg}((A \cup B) \cap H)$ is maximal. Since $A \cup B$ spans $\mathbb{P}^{3}$ we have $e_{1} \geq d+2$. First assume $h^{1}\left(H, \mathcal{I}_{H \cap(A \cup B)}(d)\right)>0$. Since $e_{1} \leq z_{1} \leq 2 d$, 6. Lemma 34], gives the existence of a line $L \subset H$ such that $\operatorname{deg}(L \cap(A \cup B)) \geq d+2$. Since $\operatorname{deg}(R \cap(A \cup B))=d+1$, we have $L \neq R$. Since the scheme $L \cup R$ has degree 1 and $L \cup R \subset H$, we get $e_{1} \geq(d+2)+(d+1)-1$. Hence $z_{1}>2 d$, a contradiction. Now assume $h^{1}\left(H, \mathcal{I}_{H \cap(A \cup B)}(d)\right)=0$. The residual exact sequence (9) gives $h^{1}\left(\mathcal{I}_{\operatorname{Res}_{H}(A \cup B)}(d-\right.$ $1))>0$. We have $\operatorname{deg}\left(\operatorname{Res}_{H}(A \cup B)\right)=\operatorname{deg}(A \cup B)-e_{1} \leq 2 d-1$. Hence there is a line $D \subset \mathbb{P}^{3}$ such that $\operatorname{deg}\left(\operatorname{Res}_{H}(A \cup B) \cap D\right) \geq d+1$. Since $B$ is reduced and $\operatorname{Res}_{H}(B) \cap D \neq \emptyset$, we have $D \nsubseteq H$ and in particular $D \neq R$. For any $o \in \operatorname{Res}_{H}(B) \cap D$ set $N_{o}:=\langle R \cup\{o\}\rangle$. Since $R \subset H$ and $o \notin H$, we have $o \notin R$. Hence $N_{o}$ is a plane. We have $\operatorname{deg}\left(N_{o} \cap(A \cup B)\right) \geq d+2$. If $h^{1}\left(N_{o}, \mathcal{I}_{N_{o} \cap(A \cup B)}(d)\right)>0$, then as above we get a contradiction. Hence we may assume $h^{1}\left(N_{o}, \mathcal{I}_{N_{o} \cap(A \cup B)}(d)\right)=0$. A residual exact sequence gives $h^{1}\left(\mathcal{I}_{\operatorname{Res}_{N_{o}}(A \cup B)}(d-1)\right)>0$. Since $\operatorname{deg}\left(\operatorname{Res}_{N_{o}}(A \cup B)\right) \leq 2 d-1$, 6, Lemma 34], gives the existence of a line $L_{o} \subset N_{o}$ such that $\operatorname{deg}\left(\operatorname{Res}_{N_{o}}(A \cup B) \cap L_{o}\right) \geq d+1$. We have $N_{o} \cap N_{o^{\prime}}=R$ for all $o^{\prime} \notin N_{o}$. Set $\alpha:=\sharp(B \cap R)$. Since $\operatorname{deg}(A \cap T) \leq 2$ for all lines $T$, we have $\alpha \geq d-1$. We get $\operatorname{deg}(A \cup B) \geq \operatorname{deg}((A \cup B) \cap R)+\alpha d \geq d+1+(d-1) d$, a contradiction.

(f) Assume $z=3$. Since $x_{1} \geq x_{2} \geq x_{3} \geq d$, we get $x_{2}=x_{3}=d, x_{1} \leq d+1$ and the existence of a line $R \subset M_{3}$ such that $E_{3} \cap M_{3} \subset R$. Since $M_{3}$ is a plane for which $\operatorname{deg}\left(E_{3} \cap M_{3}\right)$ is maximal, while there is a pencil of planes containing $R$, we have $E_{3} \subset M_{3}$ and $E_{4}=\emptyset$. Now 
instead of $M_{2}$ we take a plane $M_{2}^{\prime}$ containing $R$ and at least another point of $B \backslash B \cap M_{1}$. Since $\operatorname{deg}\left(M_{2}^{\prime} \cap E_{2}\right) \geq d+1$, we have $x_{2} \geq d+1$, a contradiction.

Therefore we may conclude that $r_{X_{3, d}}(P)=3 d-2$.

Remark 9. Fix $P \in \sigma_{4}\left(X_{m, d}\right) \backslash \sigma_{3}\left(X_{m, d}\right), m \geq 3$ and $d \geq 3$, for which $A \subset \mathbb{P}^{m}$ and hence $Z \subset X_{m, d}$ are as in Proposition 10. Here we want to describe and produce algorithmically several sets of points $S \subset X_{m, d}$ evincing $r_{X_{m, d}}(P)$.

Fix any 3-dimensional linear subspace $M$ of $\mathbb{P}^{m}$ containing $A$ and any smooth rational normal curve $T$ of $M$ such that $A \subset T$. Set $Y:=\nu_{m, d}(T)$. Thus $Y$ is a degree $3 d$ rational normal curve in its linear span. Since $Z \subset Y$, we have $P \in\langle Y\rangle$. Since $\operatorname{deg}(Z)=4$ and $Z$ is contained in a rational normal curve, we have $r_{Y}(P)=3 d-2$ (see [12] or [24, Theorem 4.1]). Hence $r_{Y}(P)=r_{X_{m, d}}(P)$. Hence any $S \subset Y$ evincing $r_{Y}(P)$ evinces $r_{X_{m, d}}(P)$. Sylvester's algorithm produces one such set $S$ (see [12, [6], [14, 7]).

Lemma 9. Fix $O \in \mathbb{P}^{3}$. Let $A$ be a 0-dimensional scheme of degree 4 such that $\operatorname{deg}(A)=4$, $A_{\text {red }}=O,\langle A\rangle=\mathbb{P}^{3}$ and $A$ is not curvilinear. Then $A$ is the first infinitesimal neighborhood of $O$ in $\mathbb{P}^{3}$ and $\left\langle\nu_{3, d}(A)\right\rangle \subset \sigma_{2}\left(X_{3, d}\right)$.

Proof. Since $A$ is not curvilinear and $\operatorname{deg}(A)=\operatorname{dim}(\langle A\rangle)+1, A$ is not as in case III of [18, Theorem 1.3]. Hence [18, Theorem 1.3], gives that $A$ is the first infinitesimal neighborhood of $O$ in $\mathbb{P}^{3}$. Since $A$ is the first infinitesimal neighborhood of $O$ in $\mathbb{P}^{3}$, every point of $\left\langle\nu_{3, d}(A)\right\rangle$ is contained in the tangent developable of $X_{3, d}$ and hence in $\sigma_{2}\left(X_{3, d}\right)$.

Proposition 11. Let $A_{1} \subset \mathbb{P}^{3}$ be a degree 3 connected 0-dimensional scheme contained in a smooth conic. Fix $O \in \mathbb{P}^{3} \backslash\left\langle A_{1}\right\rangle$ and set $A:=A_{1} \sqcup\{O\}$. Then

$$
r_{X_{3, d}}(P)=2 d
$$

for every $P \in\left\langle\nu_{3, d}(A)\right\rangle \backslash\left(\sigma_{4}^{0}\left(X_{3, d}\right) \cup \sigma_{3}\left(X_{3, d}\right)\right)$.

Proof. Assume $r_{X_{3, d}}(P) \leq 2 d-1$ and take $B \subset \mathbb{P}^{3}$ such that $\nu_{3, d}(B)$ evinces $r_{X_{3, d}}(P)$. Set $Q:=\left(A_{1}\right)_{\text {red }}$. We may repeat verbatim the proof of Proposition 9, except the last part of step (b): the case $\operatorname{Res}_{M}(A) \cap \operatorname{Res}_{M}(B) \neq \emptyset$, i.e. $B \backslash B \cap M$ contains at least one of the points $O, Q$. We may also assume $\operatorname{deg}(M \cap(A \cup B)) \leq 2 d+1$ and $h^{1}\left(\mathcal{I}_{\operatorname{Res}_{M}(A \cup B)}(d-1)\right)=0$. Since $\operatorname{deg}(B \cap L) \leq d+1$, we have $Q \in L$. Hence $Q \in M$ and $Q \notin \operatorname{Res}_{M}(B)$ even if $Q \in B$. Hence we may assume $O \notin M$ and $O \in B$. Set $B_{1}:=B \backslash\{O\}$. Notice that $\left.\left(B_{1} \backslash B_{1} \cap L\right) \cap A_{1}\right)=\emptyset$. we have $h^{1}\left(M, \mathcal{I}_{\operatorname{Res}_{L}\left(\operatorname{Res}_{L}((A \cup B) \cap M)\right.}(d-1)\right)=0$. Hence $h^{1}\left(M, \mathcal{I}_{M \cap(A \cup B)}(d)\right)=h^{1}\left(L, \mathcal{I}_{L \cap(A \cup B)}(d)\right)$. Since $h^{1}\left(\mathcal{I}_{\operatorname{Res}_{M}(A \cup B)}(d-1)\right)=0$, we get $h^{1}\left(\mathcal{I}_{A \cup B}(d)\right)=h^{1}\left(L, \mathcal{I}_{L \cap(A \cup B)}(d)\right)$. Since $L \cap A=L \cap A_{1}$ and $B \cap L=B \cap L_{1}$, we also get $h^{1}\left(\mathcal{I}_{A_{1} \cup B_{1}}(d)\right)=h^{1}\left(L, \mathcal{I}_{L \cap\left(A_{1} \cup B_{1}\right)}(d)\right)$. Since $\left.\left(B_{1} \backslash B_{1} \cap L\right) \cap A_{1}\right)=\emptyset$, as in the first proof of lemma 5 we get $\left\langle\nu_{3, d}\left(A_{1}\right)\right\rangle \cap\left\langle\nu_{d}\left(B_{1}\right)\right\rangle=\left\langle\nu_{3, d}\left(A_{1} \cap L\right)\right\rangle \cap\left\langle\nu_{d}\left(B_{1} \cap L\right)\right\rangle$. Since $h^{1}\left(\mathcal{I}_{A \cup B}(d)\right)=h^{1}\left(\mathcal{I}_{A_{1} \cup B_{1}}(d)\right)$, Grassmann's formula gives that $\left\langle\nu_{3, d}(A)\right\rangle \cap\left\langle\nu_{d}(B)\right\rangle$ is spanned by its subspaces $\left\langle\nu_{3, d}\left(A_{1}\right)\right\rangle \cap\left\langle\nu_{d}\left(B_{1}\right)\right\rangle$ and $\nu_{3, d}(O)$. Since $\operatorname{deg}\left(A_{1} \cap L\right) \leq 2$, we get $P \in \sigma_{3}\left(X_{3, d}\right)$, a contradiction.

\section{Proof of the main theorem}

Remark 10. Fix a 0 -dimensional scheme $A \subset \mathbb{P}^{m}$ of degree 4 and set $s:=\operatorname{dim}(\langle A\rangle)$. We have $s \leq \min \{m, 3\}$. Set $W:=\nu_{m, d}(\langle A\rangle)$. Hence $W$ is projectively equivalent to $X_{s, d}$. Fix any $P \in\left\langle\nu_{m, d}(A)\right\rangle$. We have $r_{X_{m, d}}(P)=r_{W}(P)$ (25, Proposition 3.1]) and any $S \subset X_{m, d}$ evincing $r_{X_{m, d}}(P)$ is contained in $W$ (see [23, Exercise 3.2.2.2], or [10, Theorem 2.1], for a generalization to the non-symmetric case). Hence not only to prove Theorem 1 it is sufficient to do the case $m \leq 3$, but any way of producing a set evincing $r_{X_{m, d}}(P)$ must work (implicitly or explicitly) inside $\langle A\rangle$. 
Proof of Theorem 1 We want to classify the $X$-rank of a point $P \in \sigma_{4}(X) \backslash \sigma_{3}(X)$ where $X$ is the Veronese embedding of $\mathbb{P}^{m}$ into $\mathbb{P}^{n}$ with $n=\left(\begin{array}{c}m+d \\ d\end{array}\right)$.

Now $\sigma_{4}(X) \backslash \sigma_{3}(X)$ is the union of

$$
\sigma_{4}^{0}(X) \backslash \sigma_{3}(X)=\left\{P \in \sigma_{4}(X) \mid r_{X}(P)=4\right\}
$$

(the set $\sigma_{4}^{0}(X)$ is defined in (6) ) and

$$
\sigma_{4}(X) \backslash\left(\sigma_{4}^{0}(X) \cup \sigma_{3}(X)\right)=\left\{P \in \sigma_{4}(X) \mid r_{X}(P)>4\right\} .
$$

We have obviously to study only the $X$-rank of points $P \in \sigma_{4}(X) \backslash\left(\sigma_{4}^{0}(X) \cup \sigma_{3}(X)\right)$. In order to do that, as already showed in Section [1, we have to study the $X$-rank of points belonging to the span of a 0-dimensional non-reduced smoothable and Gorenstein sub-scheme $Z \subset X$ of degree 4 evincing the $X$-border rank of such a point $P \in \sigma_{4}(X) \backslash\left(\sigma_{3}(X) \cup \sigma_{4}^{0}(X)\right)$ (as in Notation [1).

By Remark 10 we may restrict our attention to the case $m \leq 3$. Therefore we study separately the cases $m=1,2,3$ (we will do them in the following items (I), (II) and (III) respectively).

(I) Assume $\mathbf{m}=1$. In this case $Z=\nu_{m, d}(A)$ for $A$ contained in a line $L \subset \mathbb{P}^{m}$, hence $r_{X_{m, d}}(P)=r_{\nu_{m, d}(L)}(P)=d-2($ for [12], 6], 14], 7] or [24, Theorem 4.1]).

This case (I) proves that the set $\sigma_{4, d-2}\left(X_{m, d}\right)$ has to appear in all cases of the statement of the Theorem where $d-2 \geq 4$ (ie. cases (a), (e) and (i) of Theorem 1 moreover, for the case (a) there are no other cases to consider).

(II) Assume $\mathbf{m}=\mathbf{2}$. The scheme $A$ now is a 0 -dimensional scheme of degree 4 that is contained in a plane but not in a line (otherwise we are again in case (I)), hence it can intersect at least one line in degree 3 or it does not exist any line that intersects $A$ in degree 3 .

(II1) If $\operatorname{deg}(A \cap L)=3$ for at least one line $L \subset \mathbb{P}^{m}$ then we distinguish the following cases:

(II1.1) If $\operatorname{Res}_{L}(A) \notin L$ then $r_{X_{m, d}}(P)=d$ by Proposition 3 .

(II1.2) If $\operatorname{Res}_{L}(A) \in L$ then we study the cardinality of the support of the scheme $A$.

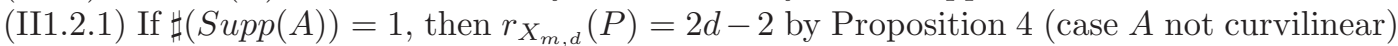
and Lemma 7 (case $A$ curvilinear).

(II1.2.2) If $\sharp(\operatorname{Supp}(A))=2$, then either $A$ is the union of two non-reduced 0-dimensional schemes both of degree 2 or $A$ is the union of a simple point $O$ and a first infinitesimal neighborhood of another point $Q \in \mathbb{P}^{2}$. In the first case $r_{X_{m, d}}(P)=2 d-2$ by Lemma [6, in the second case we have that $P \in\left\langle O, T_{\nu_{2, d}(Q)} X\right\rangle$, but since $T_{Q} X \subset \sigma_{2}(X)$, then $P \in \sigma_{3}\left(X_{m, d}\right)$.

(II1.2.3) If $\sharp(S u p p(A))=3$, then $r_{X_{m, d}}(P)=d+2$ by Proposition 5 (if $d \geq 4$ ).

(II2) Now assume that $\operatorname{deg}(A \cup L)<3$ for all lines $L$ 's contained in $\mathbb{P}^{m}$ and $\operatorname{dim}(\langle A\rangle)=2$.

(II2.1) If the generic conic through $A$ is smooth, then, by Proposition $6 r_{X_{m, d}}(P)=2 d-2$, except if $\sharp\left(A_{r e d}\right)=3$; in the latter case $r_{X_{m, d}}(P)=d+2$ by Proposition 7 (if $d \geq 4$ ).

(II2.2) If the generic conic through $A$ is not smooth, then $r_{X_{m, d}}(P)=2 d-2$ by Lemma 8 and Proposition 8 .

This case (II) proves that the subsets $\sigma_{4, d}\left(X_{m, d}\right)$ and $\sigma_{4, d+2}\left(X_{m, d}\right)$ have to appear in all cases of the statement of the Theorem where $d \geq 4$ and $m \geq 2$; the subset $\sigma_{4,2 d-2}\left(X_{m, d}\right)$ has to appear in all cases where $2 d-2 \geq 4$ and $m \geq 2$. This completes the proofs of the cases (c), (d) and (e) of the statement of Theorem 1 . Observe that the case (b) is covered by [6, Theorem 40].

(III) Assume $\mathbf{m}=\mathbf{3}$.

(III1) If $\sharp(\operatorname{Supp}(A))=1$ we may assume that $A$ is not the first infinitesimal neighborhood of a point $Q \in \mathbb{P}^{3}$, otherwise $P \in \sigma_{2}\left(\nu_{m, d}(\langle A\rangle)\right) \subset \sigma_{2}\left(X_{m, d}\right)$. Remark 9 and Proposition 10 give $r_{X m, d}(P)=3 d-2$.

(III2) If $\sharp(\operatorname{Supp}(A))=2$ we may have the following cases.

(III2.1) The scheme $A$ is the union of a simple point $O$ and a 0 -dimensional scheme $A^{\prime}$ of degree 3 supported on a point $Q \subset \mathbb{P}^{3}$ such that $\operatorname{dim}\left(\left\langle A^{\prime}\right\rangle\right)=2$ and $\left\langle\nu_{3, d}\left(A^{\prime}\right)\right\rangle \subset T_{\nu_{3, d}(Q)} X$. Therefore $P \in\left\langle O, T_{\nu_{3, d}(Q)} X\right\rangle \subset\left\langle O, \sigma_{2}(X)\right\rangle \subset \sigma_{3}(X)$. 
(III2.2) The scheme $A$ is the union of two non-reduced 0-dimensional schemes both of degree 2. Since $\langle A\rangle=\mathbb{P}^{3}$ we are in the case of Proposition 9 where we get that $r_{X_{m, d}}(P)=2 d$.

(III2.3) The scheme $A$ is the union of a simple point and of a degree 3 curvilinear 0 -dimensional scheme supported on one point. Proposition 11 gives us that $r_{X_{m, d}}(P)=2 d$.

(III3) If $\sharp(\operatorname{Supp}(A))=3$ then $A$ can only be the union of two simple points and a degree 2 non-reduced scheme. By Remark 4 we have that $r_{X_{m, d}}(P)=d+2$.

This proves that the sets $\sigma_{4,3 d-2}\left(X_{m, d}\right)$ and $\sigma_{4,2 d}\left(X_{m, d}\right)$ have to appear in all cases of the statement of the Theorem where $d \geq 3$ and $m \geq 3$. This completes the proof of the cases (f), (g), (h) and (i) of the statement of Theorem 1

\section{REFERENCES}

[1] Albera, L., Chevalier, P., Comon, P., Ferreol, A.: On the virtual array concept for higher order array processing. IEEE Trans. Sig. Proc. 53(4), 1254-1271 (2005)

[2] Alexander, J., Hirschowitz, A.: Polynomial interpolation in several variables. J. Algebraic Geom. 4(2), 201-222 (1995)

[3] Alexander, J., Hirschowitz, A.: An asymptotic vanishing theorem for generic unions of multiple points. Invent. Math. 140(2), 3003-325 (2000)

[4] Ballico, E., Bernardi, A.: Decomposition of homogeneous polynomials with low rank. Math. Z. 271 (2012), no. 3-4, 1141-1149; DOI : 10.1007/s00209-011-0907-6

[5] Brambilla, M. C., Ottaviani, G.: On the Alexander Hirschowitz theorem. J. Pure Appl. Algebra 212(5) 1229$1251(2008)$

[6] Bernardi, A., Gimigliano, A., Idà, M.: Computing symmetric rank for symmetric tensors. J. Symbolic. Comput. 46(1), 34-53 (2011)

[7] Brachat, J., Comon, P., Mourrain, B., Tsigaridas, E. P.: Symmetric tensor decomposition. Linear Algebra Appl. 433(11-12), 1851-1872 (2010)

[8] Buczyńska, W., Buczyński, J.: Secant varieties to high degree veronese reembeddings, catalecticant matrices and smoothable Gorenstein schemes. arXiv:1012.3562 4 [math.AG], J. Algebraic Geom. (to appear)

[9] Buczyński, J., Ginensky, A., Landsberg, J. M.: Determinantal equations for secant varieties and the Eisenbud-Koh-Stillman conjecture. arXiv:1007.0192 v [math.AG], J. London Math. Soc. (to appear); doi:10.1112/jlms/jds073

[10] Buczyński, J., Landsberg, J. M.: Rank of tensors and a generalization of secant varieties. Linear Algebra Appl. 438(2) 668-689 (2013)

[11] Chevalier, P.: Optimal separation of independent narrow-band sources - concept and performance. Signal Processing, Elsevier, 73, (1999), 27-48. special issue on blind separation and deconvolution

[12] Comas, G., Seiguer, M.: On the rank of a binary form. Found. Comp. Math. 11(1), 65-78 (2011)

[13] Comon, P.: Independent Component Analysis. In J-L. Lacoume, editor, Higher Order Statistics, (1992), 29-38. Elsevier, Amsterdam, London

[14] Comon, P., Golub, G. H., Lim, L.-H., Mourrain, B.: Symmetric tensors and symmetric tensor rank. SIAM J. Matrix Anal. 30(3), 1254-1279 (2008)

[15] De Lathauwer, L., Castaing, J.: Tensor-based techniques for the blind separation of ds-cdma signals. Signal Processing 87(2), 322-336 (2007)

[16] Dogăn, M. C., Mendel, J. M.: Applications of cumulants to array processing. I. aperture extension and array calibration. IEEE Trans. Sig. Proc. 43(5), 1200-1216 (1995)

[17] Eisenbud, D.: Commutative Algebra. With a view toward algebraic geometry. Graduate Texts in Mathematics, 150. Springer-Verlag, New York, 1995

[18] Eisenbud, D., Harris, J.: Finite projective schemes in linearly general position. J. Algebraic Geom. 1(1), 15-30 (1992)

[19] Fulton, W.: Algebraic Curves. Reprint of 1969 original. Advanced Book Classics. Addison-Wesley Publishing Company, Advanced Book Program, Redwood City, CA, 1989

[20] Jiang, T., Sidiropoulos, N. D.: Kruskal's permutation lemma and the identification of CANDECOMP/PARAFAC and bilinear models. IEEE Trans. Sig. Proc. 52(9), 2625-2636 (2004)

[21] Kanev, V.: Chordal varieties of Veronese varieties and catalecticant matrices. Algebraic geometry, 9, J. Math. Sci. (New York) 94(1) (1999) 1114-1125

[22] Kleppe, J.: Representing a homogeneous polynomial as a sum of powers of linear forms. Thesis for the degree of Candidatum Scientiarum, Department of Mathematics, Univ. Oslo, 1999 
[23] Landsberg, J. M.: Tensors: Geometry and Applications. Graduate Studies in Mathematics, Vol. 128, Amer. Math. Soc. Providence, 2012

[24] Landsberg, J. M., Teitler, Z.: On the ranks and border ranks of symmetric tensors. Found. Comput. Math. 10(3), 339-366 (2010)

[25] Lim, L.-H, de Silva, V.: Tensor rank and the ill-posedness of the best low-rank approximation problem. SIAM J. Matrix Anal. Appl. 30(3) 1084-1127 (2008)

[26] McCullagh, P.: Tensor Methods in Statistics. Monographs on Statistics and Applied Probability. Chapman and Hall, 1987

[27] Ottaviani, G.: An invariant regarding Waring's problem for cubic polynomials. Nagoya Math. J. 193, 95-110 (2009)

Dept. of Mathematics, University of Trento, 38123 Povo (TN), Italy

University of Turin, Department of Mathematics "Giuseppe Peano", Via Carlo Alberto 10, I-10123 (TO), Italy.

E-mail address: ballico@science.unitn.it, alessandra.bernardi@unito.it 